\title{
The Guillemin-Sternberg conjecture for noncompact groups and spaces
}

\author{
P. Hochs \& N.P. Landsman \\ Radboud University Nijmegen \\ Institute for Mathematics, Astrophysics, and Particle Physics \\ Toernooiveld 1, 6525 ED NIJMEGEN, THE NETHERLANDS \\ hochs@math.ru.nl, landsman@math.ru.nl
}

August 22, 2018

\begin{abstract}
The Guillemin-Sternberg conjecture states that "quantisation commutes with reduction" in a specific technical setting. So far, this conjecture has almost exclusively been stated and proved for compact Lie groups $G$ acting on compact symplectic manifolds, and, largely due to the use of $\operatorname{Spin}^{c}$ Dirac operator techniques, has reached a high degree of perfection under these compactness assumptions. In this paper we formulate an appropriate GuilleminSternberg conjecture in the general case, under the main assumptions that the Lie group action is proper and cocompact. This formulation is motivated by our interpretation of the "quantisation commuates with reduction" phenomenon as a special case of the functoriality of quantisation, and uses equivariant $K$-homology and the $K$-theory of the group $C^{*}$-algebra $C^{*}(G)$ in a crucial way. For example, the equivariant index - which in the compact case takes values in the representation ring $R(G)$ - is replaced by the analytic assembly map - which takes values in $K_{0}\left(C^{*}(G)\right)$ - familiar from the Baum-Connes conjecture in noncommutative geometry. Under the usual freeness assumption on the action, we prove our conjecture for all Lie groups $G$ having a discrete normal subgroup $\Gamma$ with compact quotient $G / \Gamma$, but we believe it is valid for all unimodular Lie groups.
\end{abstract}




\section{Contents}

\begin{tabular}{lll}
\hline & Introduction & 2
\end{tabular}

$\begin{array}{lll}2 & \text { Assumptions and result } & 10\end{array}$

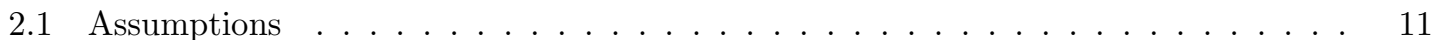

2.2 The Dirac operaton . . . . . . . . . . . . . . . . . . . . . . . . . . 13

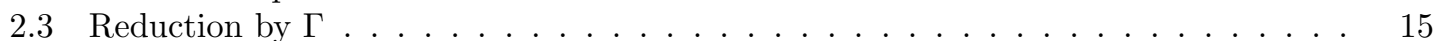

2.4 The main result . . . . . . . . . . . . . . . . . . . . . . . . . . 16

\begin{tabular}{|lll}
3 & Differential operators on vector bundles & 17
\end{tabular}

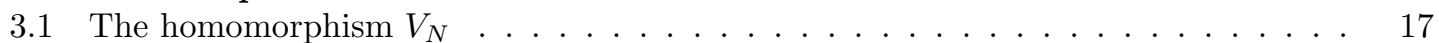

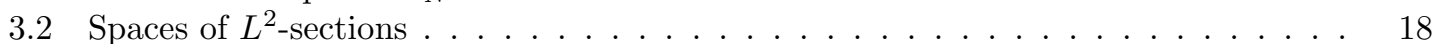

3.3 Differential operators . . . . . . . . . . . . . . . . . . . . . 20

3.4 Multiplication of sections bv functions . . . . . . . . . . . . . . . . 24

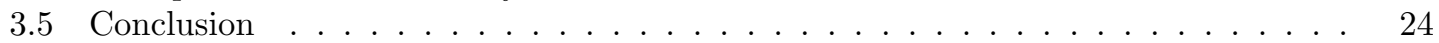

4 Dirac operators 25

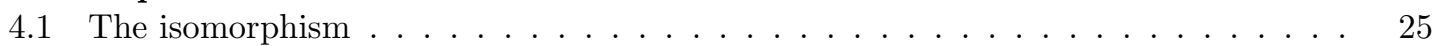

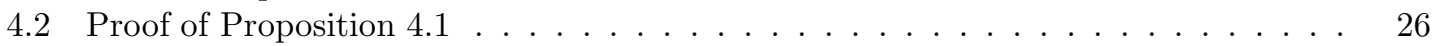

$\begin{array}{lll}5 \text { Abelian discrete groups } & 29\end{array}$

5.1 The assemblv map for abelian discrete grouns . . . . . . . . . . . . . . . . . . . . . . . . 29

5.2 The Hilbert $C^{*}$-module part of the assemblv map . . . . . . . . . . . . . . . . . . 31

5.3 The operator part of the assembly map . . . . . . . . . . . . . . . 33

5.4 Reduction . . . . . . . . . . . . . . . . . . . . . . . . . 34

6 Example: action of $\mathbb{Z}^{2 n}$ on $\mathbb{R}^{2 n} \quad 35$

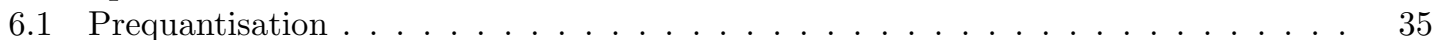

6.2 The Dirac operator . . . . . . . . . . . . . . . . . . . . . . . . 36

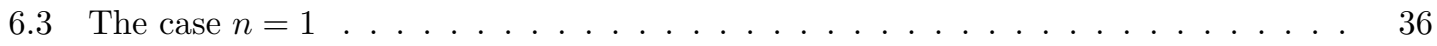

\begin{tabular}{|ll}
\hline A Naturality of the assembly map & 38
\end{tabular}

A.1 The statement . . . . . . . . . . . . . . . . . . . . . . . 38

A.2 Integrals of families of operators $\ldots \ldots \ldots \ldots \ldots \ldots$

\section{Introduction}

In 1982 two fascinating conjectures appeared about group actions. Guillemin and Sternberg 26 gave a precise mathematical formulation of Dirac's idea that "quantisation commutes with reduction" [13, in which they defined the former as geometric quantisation. As it stood, their conjecture - which they proved under special assumptions - only made sense for actions of compact Lie groups on compact symplectic manifolds. These compactness assumptions were left in place throughout all later refinements in the formulation of the conjecture and the ensuing proofs thereof under more general assumptions [32, 49, 50, 51, 53, 69, 71]. Baum and Connes, on the other hand, formulated a conjectural description of the $K$-theory of the reduced $C^{*}$-algebra $C_{r}^{*}(G)$ of a locally compact group $G$ in terms of its proper actions 4 . Here the emphasis was entirely on the noncompact case, as the Baum-Connes conjecture is trivially satisfied for compact groups. The modern formulation of the conjecture in terms of the equivariant $K$-homology of the classifying space for proper $G$ actions was given in [5]. In this version it has now been proved for all (almost) connected groups $G$ [10] as well as for a large class of discrete groups (see [70]). 
Although the two conjectures in question have quite a lot in common - such as the central role played by index theory and Dirac operators, ${ }^{1}$ or their acute relevance to modern physics, especially the quantisation of singular phase spaces (cf. 41, 43]) - the compact/noncompact divide (and perhaps also the sociological division between the communities of symplectic geometry and noncommutative geometry) seems to have precluded much "interaction" between them. As we shall see, however, some of the ideas surrounding the Baum-Connes conjecture are precisely what one needs to generalize the Guillemin-Sternberg conjecture to the locally compact case.

Being merely desirable in mathematics, such a generalization is actually crucial for physics. As a case in point, we mention the problem of constructing Yang-Mills theory in dimension 4 this is one of the Clay Mathematics Institute Millennium Prize Problems - where the groups and spaces in question are not just noncompact, but even infinite-dimensional! Also, the work of Dirac that initiated the modern theory of constraints and reduction in mechanics and field theory was originally motivated by the problem - still open - of quantising general relativity, where again the groups and spaces are infinite-dimensional. From this perspective, the present work, in which we attempt to push the Guillemin-Sternberg conjecture beyond the compactness barrier to the locally compact situation, is merely an exiguous step in the right direction.

To set the stage, we display the usual "quantisation commutes with reduction" diagram:

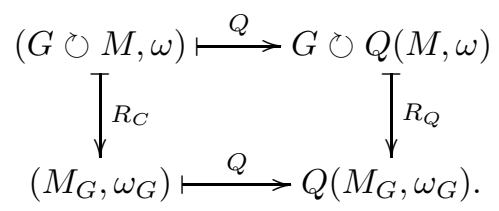

Here $(M, \omega)$ is a symplectic manifold carrying a strongly Hamiltonian action of a Lie group $G$ (cf. Section 2), with associated Marsden-Weinstein quotient $\left(M_{G}, \omega_{G}\right)$, i.e.

$$
M_{G}=\Phi^{-1}(0) / G
$$

where $\Phi: M \rightarrow \mathfrak{g}^{*}$ is the momentum map associated to the given $G$-action (cf. Subsection 2.1). This explains the term ' $R_{C}$ ' in the diagram: Classical Reduction as outlined by Dirac [13] is defined as Marsden-Weinstein reduction 11 46, 47, 48. However - and this explains both the fascination and the mystery of the field of constrained quantisation - the mathematical meaning of the remaining three arrows in the diagram is open to discussion: see [13, 18, 21, 29, 38, 66, for various perspectives. In any case, $Q$ stands for $Q$ uantization, $R_{Q}$ denotes $Q$ uantum $R$ eduction, and all authors seem to agree that, if at all possible, the arrows should be defined so as to make the diagram commute (up to isomorphism as appropriate). We will return to the significance of this commutativity requirement below, but for the moment we just remark that it by no means fixes the interpretation of the arrows.

For example, following the practice of physicists, Dirac [13] suggested that $Q(M, \omega)$ - the quantisation of the phase space $(M, \omega)$ as such - should be a Hilbert space, which subsequently is to carry a unitary representation $U$ of $G$ that "quantises" the given canonical $G$-action on $M$. We assume some procedure has been selected to construct these data; see below. Provided that $G$ is compact, the quantum reduction operation $R_{Q}$ then consists in taking the $G$-invariant part $Q(M, \omega)^{G}$ of $Q(M, \omega)$. Similarly, $Q\left(M_{G}, \omega_{G}\right)$ is a Hilbert space without any further dressing. Now assume that $M$ and $G$ are both compact: in that case, the reduced space $M_{G}$ is compact as well, so that $Q(M, \omega)$ and $Q\left(M_{G}, \omega_{G}\right)$ are typically finite-dimensional (this depends on the details of the quantisation procedure). In that case, commutativity of diagram (10) up to isomorphism just boils down to the numerical equality

$$
\operatorname{dim}\left(Q(M, \omega)^{G}\right)=\operatorname{dim}\left(Q\left(M_{G}, \omega_{G}\right)\right) .
$$

\footnotetext{
${ }^{1}$ Here the motivating role played by two closely related papers on the discrete series representations of semisimple Lie groups should be mentioned. It seems that Parthasarathy 55 influenced Bott's formulation of the GuilleminSternberg conjecture (see below), whereas Atiyah and Schmid 3 in part inspired the Baum-Connes conjecture (see 1236 ).
} 
This equality becomes a meaningful conjecture once an explicit construction of the objects $G \circlearrowright Q(M, \omega)$ and $Q\left(M_{G}, \omega_{G}\right)$ as specified above has been prescribed. Guillemin and Sternberg [26] considered the case in which the symplectic manifold $M$ is compact, prequantisable, and equipped with a positive-definite complex polarization $\mathcal{J} \subset T_{\mathbb{C}} M$ left invariant by the given strongly Hamiltonian $G$-action. Recall that a symplectic manifold $(M, \omega)$ is called prequantisable when the cohomology class $[\omega] / 2 \pi$ in $H^{2}(M, \mathbb{R})$ is integral, i.e., lies in the image of $H^{2}(M, \mathbb{Z})$ under the natural homomorphism $H^{2}(M, \mathbb{Z}) \rightarrow H^{2}(M, \mathbb{R})$. In that case, there exists a line bundle $L_{\omega}$ over $M$ whose first Chern class $c_{1}\left(L_{\omega}\right)$ maps to $[\omega] / 2 \pi$ under this homomorphism; $L_{\omega}$ is called the prequantisation line bundle over $M$. Under these circumstances, the quantisation operation $Q$ may be defined through geometric quantisation (cf. 25] for a recent and pertinent treatment): one picks a connection $\nabla$ on $L_{\omega}$ whose curvature is $\omega$, and defines the Hilbert space $Q(M, \omega)$ as the space $Q(M, \omega)=H^{0}\left(M, L_{\omega}\right)$ of polarized sections of $L_{\omega}$ (i.e. of sections annihilated by all $\left.\nabla_{X}, X \in \mathcal{J}\right)$. This Hilbert space carries a natural unitary representation of $G$ determined by the classical data, as polarized sections of $L_{\omega}$ are mapped into each other by the lift of the $G$-action. Moreover, it turns out that the reduced space $M_{G}$ - assumed to be a manifold - inherits all relevant structures on $M$ (except, of course, the $G$-action), so that it is quantisable as well, in the same fashion. Thus (3) becomes, in self-explanatory notation,

$$
\operatorname{dim}\left(H^{0}\left(M, L_{\omega}\right)^{G}\right)=\operatorname{dim}\left(H^{0}\left(M_{G}, L_{\omega_{G}}\right)\right),
$$

which Guillemin and Sternberg indeed managed to prove (see also [28]).

Impressive as this is, it is hard to think of a more favourable situation for quantisation theory then the one assumed in [26]. In the mid-1990s, various earlier attempts to generalize geometric quantisation - notably in a cohomological direction - and the associated Guillemin-Sternberg conjecture culminated in an unpublished proposal by Raoul Bott to define quantisation in terms of the (equivariant) index of a suitable Dirac operator. See, e.g., 64. As this definition forms the starting point of our generalization of the Guillemin-Sternberg conjecture to the noncompact case, we consider it in some detail. (See [16, 20, 22, 25] for the theory of $\mathrm{Spin}^{c}$ structures and the associated Dirac operators).

The first step in Bott's definition of quantisation is to canonically associate a $\operatorname{Spin}^{c}$ structure $(P, \cong)$ to a given symplectic and prequantisable manifold $(M, \omega)$ 25] 49. First, one picks an almost complex structure $J$ on $M$ that is compatible with $\omega$ (in that $\omega(\cdot, J \cdot)$ is positive definite and symmetric, i.e. a metric). This $J$ canonically induces a $\operatorname{Spin}^{c}$ structure $P_{J}$ on $T M$ [16, 25], but this is not the right one to use here. The $\operatorname{Spin}^{c}$ structure $P$ needed to quantise $M$ is the one obtained by twisting $P_{J}$ with the prequantisation line bundle $L_{\omega}$. This means (cf. [25], App. D.2.7) that $P=P_{J} \times_{\operatorname{ker}(\pi)} U\left(L_{\omega}\right)$, where $\pi: \operatorname{Spin}^{c}(n) \rightarrow S O(n)$ is the usual covering projection. We denote the associated $\operatorname{Spin}^{c}$ Dirac operator by $\not D_{M}^{L}$. See for example [16, 20. Since $M$ is even-dimensional, any Dirac operator on $M$ worth its name decomposes in the usual way as

$$
\not D=\left(\begin{array}{cc}
0 & \not D^{-} \\
\not D^{+} & 0
\end{array}\right)
$$

and we abuse notation in writing

$$
\operatorname{index}(\not D)=\operatorname{dim} \operatorname{ker}\left(\not D^{+}\right)-\operatorname{dim} \operatorname{ker}\left(\not D^{-}\right) .
$$

When $M$ is compact, the Dirac operators $\left(\not D_{M}^{L}\right)^{ \pm}$determined by the $\operatorname{Spin}^{c}$ structure $(P, \cong)$ have finite-dimensional kernels, whose dimensions define the quantisation of $(M, \omega)$ as

$$
Q(M, \omega)=\operatorname{index}\left(\not D_{M}^{L}\right) \in \mathbb{Z} .
$$

This number turns out to be independent of the choice of the $\operatorname{Spin}^{c}$ structure on $M$, as long as it satisfies the above requirement, and is entirely determined by the cohomology class $[\omega]$ (this is not true for the $\operatorname{Spin}^{c}$ structure and the associated Dirac operator itself) 25. If the symplectic manifold $(M, \omega)$ is Kähler and the line bundle $L$ is 'positive enough', then the index of $\not D_{M}^{L}$ equals 
the dimension of the space $H^{0}(M, L)$ of holomorphic sections of the prequantum line bundle. This provides some justification for Bott's definition of quantisation.

So far, quantisation just associates an integer to $(M, \omega)$. Bott's definition of quantisation gains in substance when a compact Lie group $G$ acts on $M$ in strongly Hamiltonian fashion. In that case, the pertinent $\operatorname{Spin}^{c}$ structure may be chosen to be $G$ invariant, and consequently the spaces $\operatorname{ker}\left(\not D^{ \pm}\right)$are finite-dimensional complex $G$ modules; we denote their isomorphism classes by square brackets. In this situation we write

$$
G \text {-index }(\not D)=\left[\operatorname{ker}\left(\not D^{+}\right)\right]-\left[\operatorname{ker}\left(\not D^{-}\right)\right]
$$

which defines an element of the representation $\operatorname{ring}^{2} R(G)$ of $G$. Thus the quantisation of $(M, \omega)$ with associated $G$-action may be defined as

$$
Q(G \circlearrowright M, \omega)=G \text {-index }\left(\not D_{M}^{L}\right) \in R(G) .
$$

As before, this element only depends on $[\omega]$ (and on the $G$-action, of course). When $G$ is trivial, one may identify $R(e)$ with $\mathbb{Z}$ through the isomorphism

$$
[V]-[W] \mapsto \operatorname{dim}(V)-\operatorname{dim}(W),
$$

so that (7) emerges as a special case of (9).

In this setting, the Guillemin-Sternberg conjecture makes sense as long as $M$ and $G$ are compact. Namely, in diagram (1) the upper right corner is now construed as an element of $R(G)$, whereas the lower right corner lies in $R(e) \cong \mathbb{Z}$; as in the original case, the geometric quantisation of the reduced space $\left(M_{G}, \omega_{G}\right)$ is defined whenever that of $(M, \omega)$ is. The quantum reduction map $R_{Q}: R(G) \rightarrow \mathbb{Z}$ is simply defined by

$$
R_{Q}:[V]-[W] \mapsto([V]-[W])^{G}:=\operatorname{dim}\left(V^{G}\right)-\operatorname{dim}\left(W^{G}\right),
$$

where $V^{G}$ is the $G$-invariant part of $V$, etc. Thus the Guillemin-Sternberg conjecture in the setting of Bott's definiton of quantisation simply reads

$$
\left(G-\operatorname{index}\left(\not D_{M}^{L}\right)\right)^{G}=\operatorname{index}\left(\not D_{M_{G}}^{L_{G}}\right)
$$

In this form, the conjecture was proved by Meinrenken [49, who merely assumed that $M$ and $M_{G}$ are orbifolds. ${ }^{3}$ Also see 25, 32, 53, 69, 71 for other proofs and further references. A step towards a noncompact version of the Guillemin-Sternberg conjecture has been taken by Paradan in [53], where he considers actions by compact groups on possibly noncompact manifolds. He proves that in this setting quantization commutes with reduction under certain conditions, that are met if the manifold in question is a coadjoint orbit of a semisimple Lie group, and the group acting on it is a maximal compact subgroup. Our generalisation is more or less in an orthogonal direction: we assume that the quotient of the group action is compact, rather that the group itself.

As alluded to above, using standard ideas from the context of the Baum-Connes conjecture one can formulate the Guillemin-Sternberg conjecture also for noncompact groups and manifolds. We specify our precise assumptions in Section 2 below; for the moment we just mention that it seems impossible to even formulate the conjecture unless we assume that the strongly Hamiltonian action $G \circlearrowright(M, \omega)$ is proper and cocompact (or $G$-compact, which means that $M / G$ is compact). When this is the case, we may pass from the compact to the noncompact case by making the following replacements (or lack of these) in the formalism:

1. Symplectic reduction is unchanged.

2. The definition of the $\operatorname{Spin}^{c}$ Dirac operator $\not D_{M}^{L}$ associated to $(M, \omega)$ is unchanged.

${ }^{2} R(G)$ is defined as the abelian group with one generator $[L]$ for each finite-dimensional complex representation $L$ of $G$, and relations $[L]=[M]$ when $L$ and $M$ are equivalent and $[L]+[M]=[L \oplus M]$. The tensor product of representations defines a ring structure on $R(G)$.

${ }^{3}$ Even that assumption turned out to be unnecessary [50]. 
3. The quantisation of the reduced space (which is compact by our regularity assumptions) is unchanged.

4. The representation ring $R(G)$ is replaced by $K_{0}\left(C^{*}(G)\right),{ }^{4}$ i.e. the usual $K_{0}$ group of the group $C^{*}$-algebra of $G .^{5}$

5. The equivariant index $G$-index $\left(\not D_{M}^{L}\right) \in R(G)$ is replaced by $\mu_{M}^{G}\left(\left[\not D_{M}^{L}\right]\right) \in K_{0}\left(C^{*}(G)\right)$, where

$$
\mu_{M}^{G}: K_{0}^{G}(M) \rightarrow K_{0}\left(C^{*}(G)\right)
$$

is the analytic assembly map [5] 52, 70, $K_{0}^{G}(M)$ is the equivariant analytical $K$-homology group defined by $G \circlearrowright M[5,31,34,70]$, and $\left[\not D_{M}^{L}\right]$ is the class in $K_{0}^{G}(M)$ defined by the $\operatorname{Spin}^{c}$ Dirac operator $\not D_{M}^{L}$.

6. Accordingly, the quantisation of the unreduced space $(G \circlearrowright M, \omega)$ is now given by

$$
Q(G \circlearrowright M, \omega)=G \text {-index }\left(\not D_{M}^{L}\right) \in K_{0}\left(C^{*}(G)\right),
$$

where

$$
G-\operatorname{index}\left(\not D_{M}^{L}\right):=\mu_{M}^{G}\left(\left[\not D_{M}^{L}\right]\right)
$$

purely as a matter of notation. ${ }^{6}$

7. The map $R_{Q}: R(G) \rightarrow \mathbb{Z}$ given by (10) is replaced by the map

$$
R_{Q}=\left(\int_{G}\right)_{*}: K_{0}\left(C^{*}(G)\right) \rightarrow \mathbb{Z}
$$

functorially induced by map $\int_{G}: C^{*}(G) \rightarrow \mathbb{C}$ given by $f \mapsto \int_{G} f(g) d g$ (defined on $f \in$ $L^{1}(G)$ or $f \in C_{c}(G)$ and extended to $f \in C^{*}(G)$ by continuity). ${ }^{*}$ Here we make the usual identification of $K_{0}(\mathbb{C})$ with $\mathbb{Z}$. Again, purely as a matter of notation we write this map as $x \mapsto x^{G}$.

With these replacements and the notation (14), our generalized Guillemin-Sternberg conjecture is formally given by its original version (11). More precisely:

Conjecture 1.1 (Quantisation commutes with reduction). Let $G$ be a unimodular Lie group, let $(M, \omega)$ be a symplectic manifold, and let $G \circlearrowright M$ be a proper strongly Hamiltonian action. Suppose 0 is a regular value of the associated momentum map. Suppose that the action is cocompact and admits an equivariant prequantum line bundle L. Assume there is an almost complex structure $J$ on $M$ compatible with $\omega$. Let $\not D_{M}^{L}$ be the Dirac operator on $M$ associated to $J$ and coupled to $L$, and let $\not_{M_{G}}^{L_{G}}$ be the Dirac operator on the reduced space $M_{G}$, coupled to the reduced line bundle $L_{G}$. Then

$$
\left(\int_{G}\right)_{*} \circ \mu_{M}^{G}\left[\not D_{M}^{L}\right]=\operatorname{index}\left(\not D_{M_{G}}^{L_{G}}\right) .
$$

In this paper we will prove:

Theorem 1.2. Under the assumptions listed in Subsection [2.1. Conjecture 1.1] is true.

A special case of the situation described in Subsection 2.1 is the case where $G$ is a torsion-free discrete group acting freely and cocompactly on $M$. Then Conjecture 1.1 follows from a result of Pierrot ([57], Theorème 3.3.2).

\footnotetext{
${ }^{4}$ The use of $K_{0}\left(C^{*}(G)\right)$ instead of $K_{0}\left(C_{r}^{*}(G)\right)$ is actually quite unusual in the context of the Baum-Connes conjecture; we will clarify this point in footnote $\mathbf{7}$ below.

${ }^{5}$ See $702262\left[72\right.$ for the general $K$-theory of $C^{*}$-algebras, see [14 56] for group $C^{*}$-algebras, and see [5] 63 70] for the $K$-theory of group $C^{*}$-algebras.

${ }^{6}$ This notation is justified by the fact that for $G$ and $M$ compact one actually has an equality in (14), provided one identifies $K_{0}\left(C^{*}(G)\right)$ with $R(G)$.

7 This extension would not be defined on $C_{r}^{*}(G)$ (unless $G$ is amenable). The continuous extension to $C^{*}(G)$ is a trivial consequence of the fact that $\int_{G}$ is just the representation of $C^{*}(G)$ corresponding to the trivial representation of $G$ on $\mathbb{C}$ by the usual correspondence between nondegenerate representations of $C^{*}(G)$ and continuous unitary representations of $G$ 14 56 .
} 
Example 1.3. Suppose $G$ is a semisimple Lie group with maximal compact subgroup $K$, and suppose $T \subset K$ is a maximal torus which is also a Cartan subgroup of $G$. Then by a theorem of Harish-Chandra, $G$ has discrete series representations. Let $\mathcal{O}_{\lambda} \subset \mathfrak{g}^{*}$ be the coadjoint orbit of $G$ through the element $\lambda \in \mathfrak{t}^{*}$. Then, if $i \lambda$ is a dominant integral weight, we would expect the quantisation of $\mathcal{O}_{\lambda}$, coupled to a suitable line bundle, to be the class in $K_{0}\left(C^{*}(G)\right)$ that corresponds to the discrete series representation $H_{\lambda}$ whose lowest $K$-type has highest weight $i \lambda$. In this case Conjecture 1.1 reduces to the uninteresting equality

$$
Q\left(\left(\mathcal{O}_{\lambda}\right)_{G}\right)=Q(\emptyset)=0=R_{Q}\left(Q\left(\mathcal{O}_{\lambda}\right)\right) .
$$

However, we can try to generalise Conjecture 1.1 so that the reduction map $R_{Q}$ is replaced by a reduction map $R_{Q}^{\mu}$, which amounts to taking the multiplicity of the discrete series representation whose lowest $K$-type is the dominant weight $i \mu$ instead of the multiplicity of the trivial representation. Furthermore, we note that the symplectic reduction $\left(\mathcal{O}_{\lambda}\right)^{\mu}$ of $\mathcal{O}_{\lambda}$ at the value $\mu$ is a point if $\lambda=\mu$, and empty otherwise. Therefore, we would expect that

$$
\begin{aligned}
Q\left(\left(\mathcal{O}_{\lambda}\right)^{\mu}\right) & =\delta_{\lambda \mu} \\
& =R_{Q}^{\mu}\left(H_{\lambda}\right) \\
& =R_{Q}^{\mu}\left(Q\left(\mathcal{O}_{\lambda}\right)\right),
\end{aligned}
$$

where $\delta_{\lambda \mu}$ is the Kronecker delta. The study of Conjecture 1.1 for semisimple groups is work in progress.

The truth of our generalized Guillemin-Sternberg conjecture for a special class of noncompact groups may be some justification for our specific formulation of the generalization, but in fact there is a much deeper reason why the "quantisation commutes with reduction" issue should be stated in precisely the way we have given. Namely, in the above formulation the Guillemin-Sternberg conjecture is a special case of the (conjectural) functoriality of quantisation. This single claim summarizes a research program, of which the first steps may be found in the papers [39, 40, 42. In summary, one may define a "classical" category $\mathfrak{C}$ and a "quantum" category $\mathfrak{Q}$, and construe the act of quantisation as a functor $\mathcal{Q}: \mathfrak{C} \rightarrow \mathfrak{Q}$. While the categories in question haven been rigorously constructed in [39] and [30, 33, respectively, ${ }^{8}$ the existence of the functor $\mathcal{Q}$ is so far hypothetical. However, the picture that emerges from the cases where $\mathcal{Q}$ has been constructed should hold in complete generality: deformation quantisation (in the $C^{*}$-algebraic sense first proposed by Rieffel [60, 61]) is the object side of $\mathcal{Q}$, whereas geometric quantisation (in the sense of Bott as explained above) is the arrow side of $\mathcal{Q}$. Moreover, in the setting of strongly Hamiltonian group actions as considered above, the "quantisation commutes with reduction" principle is nothing but the functoriality of quantisation (in cases where the functor has indeed been defined). ${ }^{9}$

\section{Outline of the proof}

We believe our generalized Guillemin-Sternberg conjecture to be true for all unimodular Lie groups $G$, but for reasons of human frailty we are only able to prove it in this paper when $G$ has a discrete normal subgroup $\Gamma$, such that the quotient group $K:=G / \Gamma$ is compact. ${ }^{10}$ This incorporates a number of interesting examples. Our proof is based on:

1. The validity of the Guillemin-Sternberg conjecture in the compact case 32, 49, 51, 50, 53, 69, 71;

\footnotetext{
${ }^{8}$ The objects of $\mathfrak{C}$ are integrable Poisson manifolds and its arrows are regular Weinstein dual pairs; see [39] for the meaning of the qualifiers. The arrows are composed by a generalization of the symplectic reduction procedure, and isomorphism of objects in $\mathfrak{C}$ turns out to be the same as Morita equivalence of Poisson manifolds in the sense of Xu [75]. The category $\mathfrak{Q}$ is nothing but the Kasparov-Higson category $K K$, whose objects are separable $C^{*}$-algebras and whose sets of arrows are Kasparov's $K K$-groups, composed with Kasparov's intersection product. See $70|30| 33$.

${ }^{9}$ Let $G \circlearrowright(M, \omega)$ define the Weinstein dual pair $p t \leftarrow M \rightarrow \mathfrak{g}^{*}$ in the usual way 73 , the arrow $\rightarrow$ being given by the momentum map. Functoriality of quantisation means that $\mathcal{Q}\left(p t \leftarrow M \rightarrow \mathfrak{g}^{*}\right) \times_{K K} \mathcal{Q}\left(\mathfrak{g}^{*} \hookleftarrow 0 \rightarrow p t\right)=\mathcal{Q}((p t \leftarrow$ $\left.\left.M \rightarrow \mathfrak{g}^{*}\right) \times_{C}\left(\mathfrak{g}^{*} \hookleftarrow 0 \rightarrow p t\right)\right)$. This equality is exactly the same as [1]. See 42].

${ }^{10}$ Such groups are automatically unimodular. The Guillemin-Sternberg conjecture may not hold in the nonunimodular case; see [19].
} 
2. Naturality of the assembly map for discrete groups [52];

3. Symplectic reduction in stages $38,45,48$;

4. Quantum reduction in stages.

In this paper we show, among other things, that the Guillemin-Sternberg conjecture for discrete (and possibly noncompact) groups $G$ is a consequence of the second point. For $G$ as specified in the previous paragraph, naturality of the assembly map implies a $K$-equivariant version thereof. The third and fourth items are used in an almost trivial way, namely in setting up the following diagram, which provides an outline of our proof.

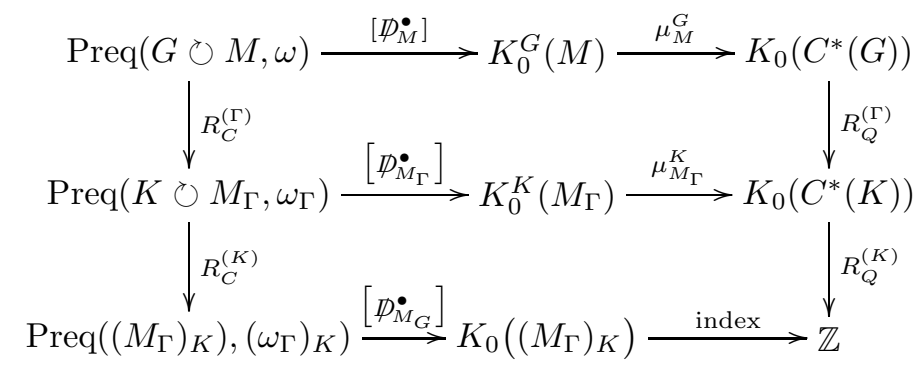

Here the following notation is used. We write

$$
\begin{aligned}
K & =G / \Gamma ; \\
M_{\Gamma} & =M / \Gamma,
\end{aligned}
$$

as $\Gamma$ is discrete (so that its associated momentum map $\Phi_{\Gamma}$ is identically zero, whence $\Phi_{\Gamma}^{-1}(0)=M$ ). Furthermore, $\operatorname{Preq}(G \circlearrowright M, \omega)$ is defined relative to a given Hamiltonian action of $G$ on a symplectic manifold $(M, \omega)$, and consists of all possible prequantisations $(L, \nabla, H)$ of this action. A necessary condition for $\operatorname{Preq}(G \circlearrowright M, \omega)$ to be nonempty is that the cohomology class $[\omega] / 2 \pi \in H^{2}(M, \mathbb{R})$ be integral. (If the group $G$ is compact, this condition is also sufficient.) We make this assumption. Similarly, Preq $\left(K \circlearrowright M_{\Gamma}, \omega_{\Gamma}\right)$ is defined given the $K$-action on $M_{\Gamma}$ induced by the $G$-action on $M$, and Preq $\left(\left(M_{\Gamma}\right)_{K},\left(\omega_{\Gamma}\right)_{K}\right)$ is just the set of prequantisations of the symplectic manifold

$$
\left(\left(M_{\Gamma}\right)_{K},\left(\omega_{\Gamma}\right)_{K}\right) \cong\left(M_{G}, \omega_{G}\right) ;
$$

this isomorphism is a special and almost trivial case of the theorem on symplectic reduction in stages [38, 45]. The maps $R_{C}^{(\Gamma)}$ and $R_{C}^{(K)}$ denote Marsden-Weinstein reduction (at zero) with respect to the groups $\Gamma$ and $K$, respectively. We define the quantum counterparts of these maps by

$$
\begin{aligned}
R_{Q}^{(\Gamma)} & :=\left(\sum_{\Gamma}\right)_{*} ; \\
R_{Q}^{(K)} & :=\left(\int_{K}\right)_{*}
\end{aligned}
$$

Here $\left(\sum_{\Gamma}\right)_{*}: K_{0}\left(C^{*}(G)\right) \rightarrow K_{0}\left(C^{*}(K)\right)$ is the map functorially induced by the map $\sum_{\Gamma}: C^{*}(G) \rightarrow$ $C^{*}(G / \Gamma)$ given by

$$
\left(\sum_{\Gamma} f\right)(\Gamma g)=\sum_{\gamma \in \Gamma} f(\gamma g)
$$

initially defined on $f \in C_{c}(G)$; see $\left[23\right.$ for the continuity of this map. ${ }^{11}$ Finally, the maps involving the symbol $\left[\not D^{\bullet}\right]$ are defined by taking the $K$-homology class of the Dirac operator coupled to a given prequantum line bundle, (as outlined above and as explained in detail in the main body of the paper below). Thus the commutativity of the upper part of diagram (16) is the equality

$$
\mu_{M_{\Gamma}}^{K}\left[\not D_{M_{\Gamma}}^{L_{\Gamma}}\right]=R_{Q}^{(\Gamma)}\left(\mu_{M}^{G}\left[\not D_{M}^{L}\right]\right)
$$

\footnotetext{
${ }^{11}$ This map can more generally be defined for any closed normal subgroup $N$ of $G$, cf. Appendix A
} 
whereas commutativity of the lower part yields

$$
\operatorname{index} \not D_{\left(M_{\Gamma}\right)_{K}}^{\left(L_{\Gamma}\right)_{K}}=R_{Q}^{(K)}\left(\mu_{M_{\Gamma}}^{K}\left[\not D_{M_{\Gamma}}^{L_{\Gamma}}\right]\right) \text {. }
$$

It is easily shown that

$$
\int_{K} \circ \sum_{\Gamma}=\int_{G},
$$

so that by functoriality of $K_{0}$ one has

$$
R_{Q}^{(K)} \circ R_{Q}^{(\Gamma)}=R_{Q}^{(G)}
$$

with $R_{Q}^{(G)}=R_{Q}$ as in (15). Using (26) and

$$
R_{C}^{(K)} \circ R_{C}^{(\Gamma)}=R_{C}^{(G)}:=R_{C},
$$

which is a mere rewriting of (19), we see that the outer diagram in (16) is equal to

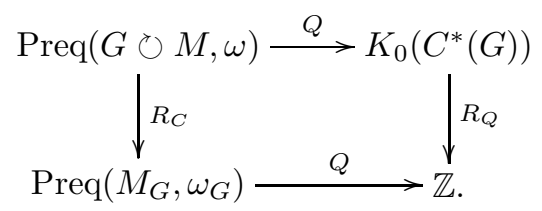

Clearly, commutativity of (28) is precisely the commutativity of diagram (1) with the post-modern meaning we have given to its ingredients. Since diagram (28) commutes when the two inner diagrams in diagram (16) commute, the latter would prove our generalized Guillemin-Sternberg conjecture. Now the lower diagram commutes by the validity of the Guillemin-Sternberg conjecture for compact $K$, whereas the upper diagram decomposes as

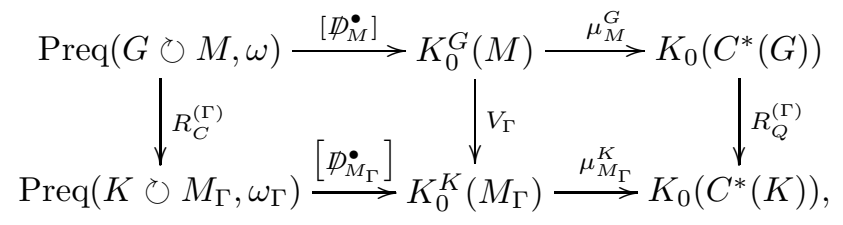

where $V_{\Gamma}$ is a map defined in Subsection 3.1 (the $V$ stands for $V$ alette, who was the first to write this map down in a more special context). Verifying the commutativity of the two inner diagrams of diagram (29), then, forms the main burden of our proof.

The commutativity of the right-hand inner diagram follows from a generalization of the naturality of the assembly map for discrete groups as proved by Valette [52] to possibly nondiscrete

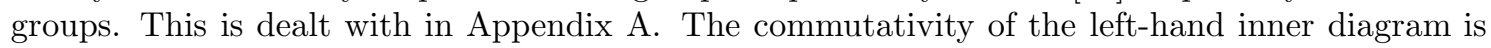
Theorem 2.9

$$
V_{\Gamma}\left[\not D M_{M}^{L}\right]=\left[\not D M_{\Gamma}^{L_{\Gamma}}\right] .
$$

The proof of this result occupies Sections 3 and 4

In Section 3 we compute the image under the map $V_{\Gamma}$ of a $K$-homology class associated to a general equivariant, elliptic, symmetric, first order differential operator $D$ on a $\Gamma$-vector bundle $E$ over a $\Gamma$-manifold $M$. If the action of $\Gamma$ on $M$ is free, as we assume, then the quotient space $E / \Gamma$ defines a vector bundle over $M / \Gamma$. The operator $D$ induces an operator $D^{\Gamma}$ on this quotient bundle. It turns out that the homomorphism $V_{\Gamma}$ maps the class associated to $D$ to the class associated to $D^{\Gamma}$.

In Section 4 we show that if $\not D_{M}^{L}$ is the Dirac operator on a symplectic manifold $M$, coupled to a prequantum line bundle $L$, then the operator $\left(\not D_{M}^{L}\right)^{\Gamma}$ from the previous paragraph is precisely the Dirac operator on the quotient $M / \Gamma$ coupled to the line bundle $L / \Gamma$.

As an encore, in Section 5 we give an independent proof of our generalized Guillemin-Sternberg conjecture for the case that $G$ is discrete and abelian. This proof, based on a paper by Lusztig 
[44] (see also [5], pp. 242-243) gives considerable insight in the situation. It is based on an explicit computation of the image under $\mu_{M}^{\Gamma}$ of a $K$-homology class $[D]$ associated to a $\Gamma$-equivariant elliptic differential operator $D$ on a $\Gamma$-vector bundle $E$ over a $\Gamma$-manifold $M$. Because in this case $C^{*}(\Gamma) \cong C(\hat{\Gamma})$ (with $\hat{\Gamma}$ the unitary dual of $\Gamma$ ), this image corresponds to the formal difference of two equivalence classes of vector bundles over $\hat{\Gamma}$. These bundles are described as the kernel and cokernel of a 'field of operators' $\left(D_{\alpha}\right)_{\alpha \in \hat{\Gamma}}$ on a 'field of vector bundles' $\left(E_{\alpha} \rightarrow M / \Gamma\right)_{\alpha \in \hat{\Gamma}}$. The operators $D_{\alpha}$ and the bundles $E_{\alpha}$ are constructed explicitly from $D$ and $E$, respectively. The quantum reduction of the class $\mu_{M}^{\Gamma}[D]$ is the index of the operator $D_{1}$ on $E_{1} \rightarrow M / \Gamma$, where $1 \in \hat{\Gamma}$ is the trivial representation. Because $D_{1}$ is the operator $D^{\Gamma}$ mentioned above, the Guillemin-Sternberg conjecture follows from the computation in Section 4

Finally, in Section 6 we check the discrete abelian case in an instructive explicit computation. We will see that the quantisation of the action of $\mathbb{Z}^{2}$ on $\mathbb{R}^{2}$ corresponds to a certain line bundle over the two-torus $\mathbb{T}^{2}=\hat{\mathbb{Z}}^{2}$. The quantum reduction of this $K$-theory class is its rank, the integer 1. This is also the quantisation of the reduced space $\mathbb{T}^{2}=\mathbb{R}^{2} / \mathbb{Z}^{2}$, as can be seen either directly or by applying Atiyah-Singer for Dirac operators. Although this is the simplest example of GuilleminSternberg for noncompact groups and spaces, the details are nontrivial and, in our opinion, well worth spelling out.

\section{Acknowledgements}

This work is supported by N.w.O. through grant no. 616.062 .384 for the second author's 'Pionier project' Quantization, noncommutative geometry, and symmetry.

The authors would like to thank Erik van den Ban, Rogier Bos, Siegfried Echterhoff, Gert Heckman, Hervé Oyono-Oyono, John Roe, Elmar Schrohe, Alain Valette and Jan Wiegerinck for useful suggestions at various stages of this work. The authors are also indebted to the referees for several useful remarks.

\section{Assumptions and result}

We now state the assumptions under which we will prove our generalised Guillemin-Sternberg conjecture, i.e. Theorem 1.2 These assumptions are mainly used in the proof of our key intermediate result, Theorem 2.9] which is proved in Sections 3 and 4

We first fix some notation and assumptions. If $M$ is a manifold, then the spaces of vector fields and differential forms on $M$ are denoted by $\mathfrak{X}(M)$ and $\Omega^{*}(M)$, respectively. The symbol $\lrcorner$ denotes contraction of differential forms by vector fields. If the manifold $M$ is equipped with an almost complex structure, then we have the space $\Omega^{0, *}(M)$ of differential forms on $M$ of type $(0, *)$. Unless stated otherwise, all manifolds, maps and actions are supposed to be $C^{\infty}$.

If a vector bundle $E \rightarrow M$ is given (which is supposed to be complex unless stated otherwise), then the space of smooth sections of $E$ is denoted by $C^{\infty}(M, E)$. If $M$ is equipped with a measure, and the bundle $E$ carries a metric, then $L^{2}(M, E)$ the space of square integrable sections of $E$. The space of differential forms on $M$ with coefficients in $E$ is denoted by $\Omega^{*}(M ; E)$, and similarly we have the space $\Omega^{0, *}(M ; E)$ for almost complex manifolds. If $F \rightarrow M$ is another vector bundle, and $\varphi: E \rightarrow F$ is a homomorphism of vector bundles, then composition with $\varphi$ gives a homomorphism of $C^{\infty}(M)$-modules

$$
\tilde{\varphi}: C^{\infty}(M, E) \rightarrow C^{\infty}(M, F) .
$$

If a group $G$ acts on $M$, and if $E$ is a $G$-vector bundle over $M$, then we have the canonical representation of $G$ on $C^{\infty}(M, E)$ given by $(g \cdot s)(m)=g \cdot s\left(g^{-1} m\right)$, for $g \in G$ and $s \in C^{\infty}(M, E)$. A superscript ' $G$ ' denotes the subspace of $G$-invariant elements. Thus we obtain for example the vector spaces $C^{\infty}(M, E)^{G}, \Omega^{0, *}(M)^{G}$, etc. The Lie algebra of a Lie group is denoted by a lower case Gothic letter, so that for example the group $G$ has the Lie algebra $\mathfrak{g}$.

In the context of Hilbert spaces and Hilbert $C^{*}$-modules, we denote the spaces of bounded, compact and finite-rank operators by $\mathcal{B}, \mathcal{K}$ and $\mathcal{F}$, respectively. 
The spaces of continuous functions, bounded continuous functions, continuous functions vanishing at infinity and compactly supported continuous functions on a topological space $X$ are denoted by $C(X), C_{b}(X), C_{0}(X)$ and $C_{c}(X)$, respectively.

\subsection{Assumptions}

Let $(M, \omega)$ be a symplectic manifold, and let $G$ be a Lie group. Suppose that $G$ has a discrete, normal subgroup $\Gamma$, such that the quotient group $K:=G / \Gamma$ is compact. For example,

- $G=K, \Gamma=\{e\}$ with $K$ a compact Lie group,

- $G=\Gamma$ discrete,

- $G=\mathbb{R}^{n}, \Gamma=\mathbb{Z}^{n}$ so that $G / \Gamma$ is the torus $\mathbb{T}^{n}$,

or direct products of these three examples. In fact, if $G$ is connected, then the subgroup $\Gamma$ must be central, and $G$ is the product of a compact group and a vector space.

The assumption that $G / \Gamma$ is compact is not needed in the proof of Theorem 2.9] it is only made so that we can apply the Guillemin-Sternberg theorem for compact groups in diagram (16).

Suppose that $G$ acts on $M$, and that the following assumptions hold.

1. The action is proper.

2. The action preserves the symplectic form $\omega$.

3. The quotient space $M / G$ is compact.

4. The action is Hamiltonian, ${ }^{12}$ in the sense that there exists a map

$$
\Phi: M \rightarrow \mathfrak{g}^{*},
$$

that is equivariant with respect to the co-adjoint representation of $G$ in $\mathfrak{g}^{*}$, such that for all $X \in \mathfrak{g}$,

$$
\left.d \Phi_{X}=-X_{M}\right\lrcorner \omega .
$$

Here $\Phi_{X}$ is the function on $M$ obtained by pairing $\Phi$ with $X$ and $X_{M}$ is the vector field on $M$ induced by $X$.

5. The discrete subgroup $\Gamma$ acts freely on $M$, and the whole group $G$ acts freely on the level set $\Phi^{-1}(0)$.

6. The symplectic manifold $(M, \omega)$ admits a $G$-equivariant prequantisation. That is, there is a $G$-equivariant complex line bundle

$$
L \rightarrow M,
$$

equipped with a $G$-invariant Hermitian metric $H$, and a Hermitian connection $\nabla$ with curvature two-form $\nabla^{2}=2 \pi i \omega$, which is equivariant as an operator from $C^{\infty}(M, L)$ to $\Omega^{1}(M ; L)$.

7. There is a $G$-invariant almost complex structure $J$ on $T M$, such that

$$
B(\cdot, \cdot):=\omega(\cdot, J \cdot)
$$

defines a Riemannian metric on $M$.

8. The manifold $M$ is complete with respect to the Riemannian metric $B$.

\footnotetext{
${ }^{12}$ Sometimes an action is called 'Hamiltonian' as opposed to 'strongly Hamiltonian' if it admits a momentum map that is not necessarily equivariant or Poisson. We will not use this terminology; for us the word 'Hamiltonian' always means 'strongly Hamiltonian'.
} 
$A d$ 3. Because the quotient $G / \Gamma$ is compact, compactness of $M / G$ is equivalent to compactness of $M / \Gamma$. The latter assumption is used in the proof of Lemma 3.3 (where $\Gamma$ is replaced by a general closed normal subgroup $N$ ), but it is not essential. Furthermore, in the definitions of $K$-homology in [5] 52 it is assumed that the orbit spaces of the group actions involved are compact. ${ }^{13}$ Finally, compactness of $M / \Gamma$ allows us to apply the Guillemin-Sternberg conjecture for compact groups and spaces to this quotient.

Ad 4. The map $\Phi$ is called a momentum map of the action. For connected groups, the assumption that $\Phi$ is equivariant is equivalent to the assumpion that it is a Poisson map with respect to the negative Lie-Poisson structure on $\mathfrak{g}^{*}$ (see for example 38, Corollary III.1.2.5 or [46], §11.6). Note that if $G=\Gamma$ is discrete, then the action is automatically Hamiltonian. Indeed, $\operatorname{Lie}(\Gamma)=\{0\}$, so that the zero map is a momentum map.

Ad 5. Freeness of the action of $\Gamma$ on $M$ implies that the quotient $M / \Gamma$ is smooth, and that a $\Gamma$-vector bundle $E \rightarrow M$ induces a vector bundle $E / \Gamma \rightarrow M / \Gamma$. And if $G$ acts freely on $\Phi^{-1}(0)$, then it follows from de definition of momentum maps that 0 is a regular value of $\Phi$ (Smale's lemma [65]), so that the reduced space $M_{G}$ is a smooth manifold. If freeness is replaced by local freeness in Assumption 5, then $M / \Gamma$ and $M_{G}$ are orbifolds. In that case, a quotient vector bundle $E / \Gamma \rightarrow M / \Gamma$ can be replaced by 'the vector bundle over $M / \Gamma$ whose space of smooth sections is $C^{\infty}(M, E)^{\Gamma}$ (see Proposition 2.8)..$^{14}$

Ad 6. Example 2.3 below shows that it is not always obvious if this assumption is satisfied. Equivariance of the connection $\nabla$ implies that the Dolbeault-Dirac operator on $M$, coupled to $L$ via $\nabla$ (Definition 2.5 ) is equivariant.

The Kostant formula

$$
X \mapsto-\nabla_{X_{M}}+2 \pi i \Phi_{X}
$$

defines a representation of $\mathfrak{g}$ in the space of smooth sections of $L$. In the literature on the GuilleminSternberg conjecture, it is usually assumed that the action of $G$ on $L$ is such that the corresponding representation of $\mathfrak{g}$ in $C^{\infty}(M, L)$ is given by the Kostant formula. Then, if the group $G$ is connected, the connection $\nabla$ satisfies $g \nabla_{v} g^{-1}=\nabla_{g \cdot v}$ for all $g \in G$ and $v \in \mathfrak{X}(M)$. This property is equivalent to equivariance of the connection $\nabla$ in the sense of assumption 6 .

If the manifold $M$ is simply connected and the group $G$ is discrete, then Hawkins [27] gives a procedure to lift the action of $G$ on $M$ to a projective action on the trivial line bundle over $M$, such that a given connection is equivariant. Under a certain condition (integrality of a group cocycle), this projective action is an actual action.

$A d$ 7. An equivalent assumption is that there exists a $G$-invariant Riemannian metric on $M$. (See e.g. 25], pp. 111-112.)

$A d$ 8. This assumption implies that the Dirac operator on $M$ is essentially self-adjoint on its natural domain (see Subsection 2.2).

The assumptions and notation above will be used in this section and in Section 4. In Section 3 . we will work under more general assumptions.

Remark 2.1. If the group $G$ is compact, then some of these assumptions are always satisfied. First of all, the action is automatically proper. Furthermore, if the cohomology class $[\omega] \in H_{\mathrm{dR}}^{2}(M)$ is integral, then a prequantum line bundle exists, and the connection can be made equivariant by averaging over $G$. Also, averaging over $G$ makes any Riemannian metric $G$-invariant, so that assumption 7 is also satisfied. And finally, since $M / G$ is compact, so is $M$. In particular, $M$ is complete, so assumption 8 is satisfied.

Remark 2.2. If the action of $G$ on $M$ is (locally) free and Hamiltonian, and $M / G$ is compact, then $G$ must be discrete. Indeed, if the action is locally free then by Smale's lemma the momentum map $\Phi$ is a submersion, and in particular an open mapping. And since it is $G$-equivariant, it induces

$$
\Phi^{G}: M / G \rightarrow \mathfrak{g}^{*} / \operatorname{Ad}^{*}(G)
$$

\footnotetext{
${ }^{13} K$-homology can be defined more generally, but the compactness assumption makes things a little easier.

${ }^{14}$ It is not a good idea to make the stronger assumption that $G$ acts freely on $M$. For in that case, $G$ must be discrete (see Remark 2.2.
} 
which is also open. So, since $M / G$ is compact, the image

$$
\Phi^{G}(M / G) \subset \mathfrak{g}^{*} / \operatorname{Ad}^{*}(G)
$$

is a compact open subset. Because $\mathfrak{g}^{*} / \operatorname{Ad}^{*}(G)$ is connected, it must therefore be compact. This, however, can only be the case (under our assumptions) when $G$ is discrete. ${ }^{15}$ Indeed, we have

$$
\operatorname{Ad}^{*}(G) \cong \operatorname{Ad}^{*}(K) \subset G L\left(\mathfrak{k}^{*}\right) \cong G L\left(\mathfrak{g}^{*}\right) .
$$

So $\operatorname{Ad}^{*}(G)$ is compact, and $\mathfrak{g}^{*} / \operatorname{Ad}^{*}(G)$ cannot be compact, unless $\mathfrak{g}^{*}=0$, i.e. $G$ is discrete.

Example 2.3. Let $M=\mathbb{C}$, with coordinate $z=q+i p$, and the standard complex structure. We equip $M$ with the symplectic form $\omega=d p \wedge d q$.

Consider the group $G=\Gamma=\mathbb{Z}+i \mathbb{Z} \subset \mathbb{C}$. We let it act on $M$ by addition:

$$
(k+i l) \cdot z=z+k+i l,
$$

for $k, l \in \mathbb{Z}, z \in \mathbb{C}$.

Consider the trivial line bundle $L=M \times \mathbb{C} \rightarrow M$. We define an action of $\mathbb{Z}+i \mathbb{Z}$ on $L$ by letting the elements $1, i \in \mathbb{Z}+i \mathbb{Z}$ act as follows:

$$
\begin{aligned}
& 1 \cdot(z, w)=(z+1, w) ; \\
& i \cdot(z, w)=\left(z+i, e^{-2 \pi i z} w\right),
\end{aligned}
$$

for $z, w \in \mathbb{C}$. Define a Hermitian metric $H$ on $L$ by

$$
H\left((q+i p, w),\left(q+i p, w^{\prime}\right)\right)=e^{2 \pi\left(p-p^{2}\right)} w \bar{w}^{\prime} .
$$

This metric is $\mathbb{Z}+i \mathbb{Z}$-invariant, and the connection

$$
\nabla=d+2 \pi i p d z+\pi d p
$$

is Hermitian, $\mathbb{Z}+i \mathbb{Z}$-invariant, and has curvature form $2 \pi i \omega$.

The details of this example are worked out in Section [6] where we also give some motivation for these formulae.

Example 2.4. Suppose $\left(M_{1}, \omega_{1}\right)$ is a compact symplectic manifold, $K$ is a compact Lie group, and let a proper Hamiltonian action of $K$ on $M_{1}$ be given. Let $\Phi$ be the momentum map, and suppose $K$ acts freely on $\Phi^{-1}(0)$. Suppose $[\omega]$ is an integral cohomology class. (These assumptions are made for example by Tian \& Zhang [69.) Let $\Gamma$ be a discrete group acting properly and freely on a symplectic manifold $\left(M_{2}, \omega_{2}\right)$, leaving $\omega_{2}$ invariant. Suppose that $M_{2} / \Gamma$ is compact, and that there is an equivariant prequantum line bundle over $M_{2}$. Then the direct product action of $K \times \Gamma$ on $M_{1} \times M_{2}$ satisfies the assumptions of this section.

\subsection{The Dirac operator}

The prequantum connection $\nabla$ on $L$ induces a differential operator

$$
\nabla: \Omega^{k}(M ; L) \rightarrow \Omega^{k+1}(M ; L),
$$

such that for all $\alpha \in \Omega^{k}(M)$ and $s \in C^{\infty}(M, L)$,

$$
\nabla(\alpha \otimes s)=d \alpha \otimes s+(-1)^{k} \alpha \wedge \nabla s .
$$

Let $\pi^{0, *}$ be the projection

$$
\pi^{0, *}: \Omega_{\mathbb{C}}^{*}(M ; L) \rightarrow \Omega^{0, *}(M ; L) .
$$

\footnotetext{
${ }^{15}$ If $G=K$ is a compact connected Lie group, then $\mathfrak{k}^{*} / \operatorname{Ad}^{*}(K)$ is a Weyl chamber.
} 
Composing the restriction to $\Omega^{0, q}(M ; L)$ of the complexification of $\nabla$ with $\pi^{0, *}$, we obtain a differential operator

$$
\bar{\partial}_{L}:=\pi^{0, *} \circ \nabla: \Omega^{0, q}(M ; L) \rightarrow \Omega^{0, q+1}(M ; L) .
$$

Let

$$
\bar{\partial}_{L}^{*}: \Omega^{0, q+1}(M ; L) \rightarrow \Omega^{0, q}(M ; L)
$$

be the formal adjoint of $\bar{\partial}_{L}$ with respect to the $L^{2}$-inner product on compactly supported forms.

Definition 2.5 (Dolbeault-Dirac operator). The Dolbeault-Dirac operator on $M$, coupled to $L$ via $\nabla$ is the differential operator

$$
\not D_{M}^{L}:=\bar{\partial}_{L}+\bar{\partial}_{L}^{*}: \Omega^{0, *}(M ; L) \rightarrow \Omega^{0, *}(M ; L) .
$$

This operator is $G$-equivariant by equivariance of the connection $\nabla$ (assumption 6 ) and invariance of the almost complex structure $J$ (assumption 7).

The Dolbeault-Dirac operator defines an unbounded symmetric operator on the Hilbert space of $L^{2}$-sections

$$
H_{M}^{L}:=L^{2}\left(M, \bigwedge^{0, *} T^{*} M \otimes L\right),
$$

with respect to the Liouville measure $d m$ on $M$. The metric on $\bigwedge^{0, *} T^{*} M \otimes L$ comes from the given metric $H$ on $L$ and from the Riemannian metric $B=\omega(\cdot, J \cdot)$ on $T M$. Because $M$ is metrically complete, the closure of $\not D_{M}^{L}$ is a self-adjoint operator on $H_{M}^{L} \cdot{ }^{16}$ So we can apply the functional calculus (see e.g. [58]) to define the bounded operator $F_{M}^{L}:=b\left(\not D_{M}^{L}\right.$ ) on $H_{M}^{L}$, where $b$ is a normalising function [31]:

Definition 2.6. A smooth function $b: \mathbb{R} \rightarrow \mathbb{R}$ is called a normalising function if it has the following three properties:

- $b$ is odd;

- $b(t)>0$ for all $t>0$;

- $\lim _{t \rightarrow \pm \infty} b(t)= \pm 1$.

Let $C_{0}(M)$ denote the $C^{*}$-algebra of continuous functions on $M$ that vanish at infinity, and let $\mathcal{B}\left(H_{M}^{L}\right)$ be the the $C^{*}$-algebra of bounded operators on $H_{M}^{L}$. Let

$$
\pi_{M}: C_{0}(M) \rightarrow \mathcal{B}\left(H_{M}^{L}\right)
$$

be the representation of $C_{0}(M)$ on $H_{M}^{L}$ defined by multiplication of sections by functions. Then the triple $\left(H_{M}^{L}, F_{M}^{L}, \pi_{M}\right)$ defines a $K$-homology class

$$
\left[\not D_{M}^{L}\right]:=\left[H_{M}^{L}, F_{M}^{L}, \pi_{M}\right] \in K_{0}^{G}(M),
$$

which is independent of $b$. See [5, 31, 52] for the definition of $K$-homology, and in particular Theorem 10.6.5 in [31] for the claim that $\left[\not D_{M}^{L}\right]$ defines a $K$-homology class.

Remark 2.7. The Dolbeault-Dirac operator has the same principal symbol as the $\operatorname{Spin}^{c}$ Dirac operator associated to the almost complex structure $J$ on $M$ and the line bundle $L$ (see e.g. [16], page 48), namely the Clifford action of $T^{*} M$ on $\bigwedge^{0, *} T^{*} M \otimes L$. So the two operators define the same class in $K$-homology. (The linear path between the operators provides a homotopy.) Thus, if we consider the $K$-homology class $\left[\not D_{M}^{L}\right]$, we may take $\not D_{M}^{L}$ to be either the $\operatorname{Spin}^{c}$ Dirac operator or the Dolbeault-Dirac operator.

\footnotetext{
${ }^{16}$ This follows from the connection between Dirac operators and Riemannian metrics as given for example in [1], section VI.1, combined with Section 10.2 of [31]. See also [74] and page 96 of [20].
} 


\subsection{Reduction by $\Gamma$}

Because the subgroup $\Gamma$ of $G$ is discrete, the symplectic quotient $M_{\Gamma}$ of $M$ by $\Gamma$ is equal to the orbit manifold $M / \Gamma$. The symplectic form $\omega_{\Gamma}$ on $M / \Gamma$ is determined by

$$
p^{*} \omega_{\Gamma}=\omega,
$$

with $p: M \rightarrow M / \Gamma$ the quotient map. The action of $G$ on $M$ descends to an action of $G / \Gamma$ on $M / \Gamma$, which satisfies the assumptions of Section 2.1 (with $M, \omega$, and $G$ replaced by $M / \Gamma, \omega_{\Gamma}$ and $G / \Gamma$, respectively). The quotient $L / \Gamma$ turns out to be the total space of a prequantum line bundle over $M / \Gamma$. This is implied by the following fact.

Proposition 2.8. Let $H$ be a group acting properly and freely on a manifold $M$. Let $q: E \rightarrow M$ be an $H$-vector bundle. Then the induced projection

$$
q^{H}: E / H \rightarrow M / H
$$

defines a vector bundle over $M / H$.

Let $C^{\infty}(M, E)^{H}$ be the space of $H$-invariant sections of $E$. The linear map

$$
\psi_{E}: C^{\infty}(M, E)^{H} \rightarrow C^{\infty}(M / H, E / H),
$$

defined by

$$
\psi_{E}(s)(H \cdot m)=H \cdot s(m),
$$

is an isomorphism of $C^{\infty}(M)^{H} \cong C^{\infty}(M / H)$-modules.

Hence the quotient space $L / \Gamma$ is a complex line bundle over $M / \Gamma$, and its space of sections is $C^{\infty}(M / \Gamma, L / \Gamma) \cong C^{\infty}(M, L)^{\Gamma}$.

The connection $\nabla$ on $L$ is $G$-equivariant, so it defines a $G / \Gamma$-equivariant connection $\nabla^{\Gamma}$ on $L / \Gamma$ as follows. Let $p: M \rightarrow M / \Gamma$ be the quotient map. Its tangent map $T p: T M \rightarrow T(M / \Gamma)$ induces an isomorphism of vector bundles over $M / \Gamma$

$$
T p^{\Gamma}:(T M) / \Gamma \rightarrow T(M / \Gamma) .
$$

Let

$$
\widetilde{T p^{\Gamma}}: C^{\infty}(M / \Gamma,(T M) / \Gamma) \stackrel{\cong}{\rightrightarrows}(M / \Gamma)
$$

be the isomorphism of $C^{\infty}(M / \Gamma)$ modules induced by $T p^{\Gamma}$. Consider the isomorphism of $C^{\infty}(M)^{\Gamma} \cong C^{\infty}(M / \Gamma)$ modules

$$
\varphi: \mathfrak{X}(M)^{\Gamma} \stackrel{\psi_{T M}}{\longrightarrow} C^{\infty}(M / \Gamma,(T M) / \Gamma) \stackrel{\widetilde{T p^{\Gamma}}}{\longrightarrow} \mathfrak{X}(M / \Gamma) .
$$

Let $v \in \mathfrak{X}(M)^{\Gamma}$ be a $\Gamma$-invariant vector field on $M$. Then the operator $\nabla_{v}$ is $\Gamma$-equivariant, and hence maps $\Gamma$-invariant scetions of $L$ to invariant sections. The covariant derivative $\nabla_{\varphi(v)}^{\Gamma}$ on $C^{\infty}(M / \Gamma, L / \Gamma)$ is defined by the commutativity of the following diagram:

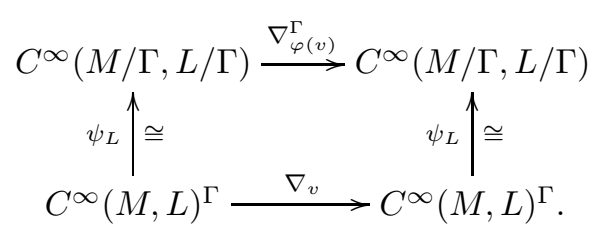

A computation shows that $\nabla^{\Gamma}$ satisfies the properties of a connection, and that its curvature is

$$
\left(\nabla^{\Gamma}\right)^{2}=2 \pi i \omega_{\Gamma} .
$$

Furthermore, the $G$-invariant Hermitian metric $H$ on $L$ descends to a $G / \Gamma$-invariant Hermitian metric $H^{\Gamma}$ on $L / \Gamma$, and the connection $\nabla^{\Gamma}$ is Hermitian with respect to this metric. Finally, the 
$G$-invariant almost complex structure $J$ on $T M$ induces a $G / \Gamma$-invariant almost complex structure $J^{\Gamma}$ on $T(M / \Gamma) \cong(T M) / \Gamma$. The corresponding Riemannian metric is denoted by

$$
B^{\Gamma}=\omega_{\Gamma}\left(\cdot, J^{\Gamma} \cdot\right) .
$$

From the Dirac operator $\not D_{M / \Gamma}^{L / \Gamma}$ on $M / \Gamma$ associated to the almost complex structure $J^{\Gamma}$, coupled to the line bundle $L / \Gamma$ via $\nabla^{\Gamma}$, we form the bounded operator $F_{M / \Gamma}^{L / \Gamma}:=b\left(\not D_{M / \Gamma}^{L / \Gamma}\right)$ (where $b$ is a normalising function) on the Hilbert space

$$
H_{M / \Gamma}^{L / \Gamma}:=L^{2}\left(M / \Gamma, \bigwedge^{0, *} T^{*}(M / \Gamma) \otimes L / \Gamma\right),
$$

which is defined with respect to the metrics on $\bigwedge^{0, *} T^{*}(M / \Gamma)$ and $L / \Gamma$ coming from those on $\wedge^{0, *} T^{*} M$ and $L$ respectively, and the measure $d \mathcal{O}$ on $M / \Gamma$ defined as follows.

Let $U \subset M$ be a fundamental domain for the $\Gamma$-action. That is, $U$ is an open subset, $\Gamma \cdot U$ is dense in $M$, and if $m$ is a point in $U$, and $\gamma \in \Gamma$ is such that $\gamma \cdot m \in U$, then $\gamma=e$. Then for all measurable functions $f$ on $M / \Gamma$ we define

$$
\int_{M / \Gamma} f(\mathcal{O}) d \mathcal{O}:=\int_{U} p^{*} f(m) d m
$$

where $p: M \rightarrow M / \Gamma$ is the quotient map. If $V$ is another fundamental domain, the subsets $\Gamma \cdot U$ and $\Gamma \cdot V$ differ by a set of measure zero, so this definition does not depend on the choice of the fundamental domain. An equivalent way of defining $d \mathcal{O}$ is to say that the $d \mathcal{O}$-measure of a measurable subset $A \subset M / \Gamma$ equals the $d m$-measure of the subset $p^{-1}(A) \cap U$ of $M$. And since $d m$ is the Liouville measure on $(M, \omega)$, the measure $d \mathcal{O}$ is precisely the Liouville measure on $\left(M / \Gamma, \omega_{\Gamma}\right)$.

We then have the $K$-homology class

$$
\left[\not D_{M / \Gamma}^{L / \Gamma}\right]:=\left[H_{M / \Gamma}^{L / \Gamma}, F_{M / \Gamma}^{L / \Gamma}, \pi_{M / \Gamma}\right] \in K_{0}^{G / \Gamma}(M / \Gamma) .
$$

\subsection{The main result}

In Subsection 3.1 we define a homomorphism

$$
V_{\Gamma}: K_{0}^{G}(M) \rightarrow K_{0}^{G / \Gamma}(M / \Gamma)
$$

such that the following diagram commutes:

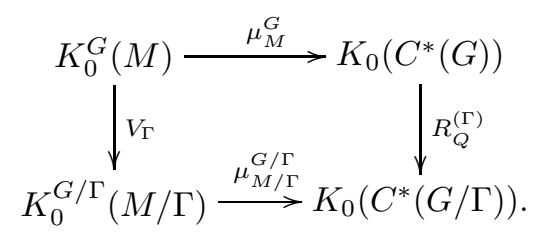

Here $\mu_{M}^{G}$ and $\mu_{M / \Gamma}^{G / \Gamma}$ are analytic assembly maps (see [5, 70, 52]), and the homomorphism $R_{Q}^{(\Gamma)}$ is defined in (20). In Appendix $\mathrm{A}$ we sketch how to generalise Valette's proof in [52] of commutativity of diagram (33) ('naturality of the assembly map') for discrete groups to the nondiscrete case.

The main step in our proof of Theorem 1.2 is the following:

Theorem 2.9. The homomorphism $V_{\Gamma}$ maps the $K$-homology class of the Dirac operator $\not_{M}^{L}$ to the $K$-homology class of the Dirac operator $\not D_{M / \Gamma}^{L / \Gamma}$ on the reduced space $M / \Gamma$ :

$$
V_{\Gamma}\left(\left[\not D_{M}^{L}\right]\right)=\left[\not D_{M / \Gamma}^{L / \Gamma}\right] \in K_{0}^{G / \Gamma}(M / \Gamma)
$$


As we noted in the Introduction, Theorem 1.2 follows from Theorem 2.9 the naturality of the assembly map (diagram (33)) and the Guillemin-Sternberg conjecture for compact $G$ and $M$.

Remark 2.10. We will actually prove a stronger result than Theorem 2.9 Write

$$
V_{\Gamma}\left(\left[H_{M}^{L}, F_{M}^{L}, \pi_{M}\right]\right)=\left[H_{\Gamma}, F_{\Gamma}, \pi_{\Gamma}\right] .
$$

Then there is a unitary isomorphism

$$
\chi: H_{\Gamma} \rightarrow H_{M / \Gamma}^{L / \Gamma}
$$

that intertwines the pertinent representations of $G / \Gamma$ and of $C(M / \Gamma)$, and the operators $F_{\Gamma}$ and $F_{M / \Gamma}^{L / \Gamma}$.

\section{Differential operators on vector bundles}

In this section, we will compute the image under the homomorphism $V_{N}$ in diagram (33) of a $K$-homology class associated to an equivariant elliptic first order differential operator on a vector bundle over a smooth manifold. The result is Corollary 3.13 In Section 4 we will see that Theorem 2.9 is a special case of Corollary 3.13]

Let $G$ be a unimodular Lie group, and let $N$ be a closed normal subgroup of $G$. Let $d g$ and $d n$ be Haar measures on $G$ and $N$ respectively. Let $M$ be a smooth manifold on which $G$ acts properly, such that the action of $N$ on $M$ is free. Suppose $M / N$ is compact. ${ }^{17}$

\subsection{The homomorphism $V_{N}$}

Let us briefly state the definition of the homomorphism

$$
V_{N}: K_{0}^{G}(M) \rightarrow K_{0}^{G / N}(M / N) .
$$

For details we refer to $\left[52\right.$. Let $H$ be a $\mathbb{Z}_{2}$-graded Hilbert space carrying a unitary representation of $G, F$ a $G$-equivariant bounded operator on $H$, and $\pi$ a representation of $C_{0}(M)$ in $H$ that is $G$-equivariant in the sense that for all $g \in G$ and $f \in C_{0}(M)$, one has $g \pi(f) g^{-1}=\pi(g \cdot f)$. Suppose $(H, F, \pi)$ defines a $K$-homology cycle. Then

$$
V_{N}[H, F, \pi]:=\left[H_{N}, F_{N}, \pi_{N}\right],
$$

with $H_{N}, F_{N}$ and $\pi_{N}$ defined as follows. ${ }^{18}$

Consider the subspace $H_{c}:=\pi\left(C_{c}(M)\right) H \subset H$ and the sesquilinear form $(\cdot, \cdot)_{N}$ on $H_{c}$ given by

$$
(\xi, \eta)_{N}:=\int_{N}(\xi, n \cdot \eta)_{H} d n
$$

for $\xi, \eta \in H_{c}$. This form turns out to be positive semidefinite. Consider the quotient space of $H_{c}$ by the kernel of this form, and complete this quotient in the inner product $(\cdot, \cdot)_{N}$. This completion is $H_{N}$.

Next, we use the fact that any $K$-homology class can be represented by a cycle whose operator is properly supported:

Definition 3.1. The operator $F$ is properly supported if for every $f \in C_{c}(M)$ there is an $h \in C_{c}(M)$ such that $\pi(h) F \pi(f)=F \pi(h)$.

Suppose $F$ is properly supported. Then it preserves $H_{c}$, and the restriction of $F$ to $H_{c}$ is bounded with respect to the form $(\cdot, \cdot)_{N}$. Hence $\left.F\right|_{H_{c}}$ induces a bounded operator $F_{N}$ on $H_{N}$ by continuous extension. The representation $\pi$ extends to the multiplier algebra $C_{b}(M)$ of $C_{0}(M)$. The algebra $C_{0}(M / N)$ can be embedded into $C_{b}(M)$ via the isomorphism $C(M / N) \cong C(M)^{N}$, and then we can use an argument similar to the one used in the definition of $F_{N}$ to show that $\pi$ induces a representation $\pi_{N}$ of $C_{0}(M / N)$ in $H_{N}$.

\footnotetext{
${ }^{17}$ Compactness of $M / N$ is used in the proof of Lemma 3.3 but not in an essential way (see Footnote 21].

${ }^{18}$ The construction below originated in Rieffel's theory of induced representations of $C^{*}$-algebras $[59$, which independently found its way into the Baum-Connes conjecture [5] and into the theory of constrained quantisation 37 38.
} 


\subsection{Spaces of $L^{2}$-sections}

Now let $q: E \rightarrow M$ be a $G$-vector bundle, equipped with a $G$-invariant metric $(\cdot, \cdot)_{E}$. Let $d m$ be a $G$-invariant measure on $M$, and let $L^{2}(M, E)$ be the space of square-integrable sections of $E$ with respect to this measure. Let $\pi_{M}: C_{0}(M) \rightarrow \mathcal{B}\left(L^{2}(M, E)\right)$ be the representation defined by multiplying sections by functions. Let $L^{2}(M, E)_{N}$ be the Hilbert space constructed from $L^{2}(M, E)$ as in the definition of the homomorphism $V_{N}$. We will show that $L^{2}(M, E)_{N}$ is naturally isomorphic ${ }^{19}$ to the Hilbert space $L^{2}(M / N, E / N)$ of square-integrable sections of the quotient vector bundle

$$
q_{N}: E / N \rightarrow M / N
$$

(see Proposition 2.8). The $L^{2}$-inner product on sections of $E / N$ is defined via the metric on $E / N$ induced by the one on $E$, and the measure $d \mathcal{O}$ on $M / N$ with the property that for all measurable sections $^{20} \varphi: M / N \rightarrow M$ and all $f \in C_{c}(M)$,

$$
\int_{M} f(m) d m=\int_{M / N} \int_{N} f(n \cdot \varphi(\mathcal{O})) d n d \mathcal{O}
$$

(see [8], Proposition 4b, p. 44). If $N$ is discrete and $d n$ is the counting measure, then the measure $d \mathcal{O}$ from (32) satisfies property (34).

Note that in this example, the space

$$
L_{c}^{2}(M, E):=\pi\left(C_{c}(M)\right) L^{2}(M, E)
$$

is the space of compactly supported $L^{2}$-sections of $E$. Consider the linear map

$$
\chi: L_{c}^{2}(M, E) \rightarrow L^{2}(M / N, E / N),
$$

defined by

$$
\chi(s)(N m):=N \cdot \int_{N} n \cdot s\left(n^{-1} m\right) d n,
$$

for all $s \in L_{c}^{2}(M, E)$ and $m \in M$. Because $s$ is compactly supported and the action is proper, the integrand is compactly supported for all $m \in M$.

Proposition 3.2. The map $\chi$ induces a natural $G / N$-equivariant unitary isomorphism

$$
\chi: L^{2}(M, E)_{N} \cong L^{2}(M / N, E / N) .
$$

Proof. It follows from a lengthy but straightforward computation that the map $\chi$ is isometric, in the sense that for all $s \in L_{c}^{2}(M, E)$,

$$
\|\chi(s)\|_{L^{2}(M / N, E / N)}=\|s\|_{N},
$$

where $\|\cdot\|_{N}$ is the norm corresponding to the inner product $(\cdot, \cdot)_{N}$. Furthermore, $\chi$ is surjective, see Lemma 3.3 below. By these two properties, $\chi$ induces a bijective linear map

$$
\chi: L_{c}^{2}(M, E) / \mathcal{K} \rightarrow L^{2}(M / N, E / N),
$$

where $\mathcal{K}$ is the space of sections $s \in L_{c}^{2}(M, E)$ with $\|s\|_{N}=0$. This map is a norm preserving linear isomorphism from $L_{c}^{2}(M, E) / \mathcal{K}$ onto the complete space $L^{2}(M / N, E / N)$. Hence the space $L_{c}^{2}(M, E) / \mathcal{K}$ is already complete, so that $L^{2}(M, E)_{N}=L_{c}^{2}(M, E) / \mathcal{K}$.

So (37) is actually a unitary isomorphism

$$
\chi: L^{2}(M, E)_{N} \rightarrow L^{2}(M / N, E / N) .
$$

The fact that $N$ is a normal subgroup implies that this isomorphism intertwines the pertinent representations of $G / N$.

\footnotetext{
${ }^{19} \mathrm{~A}$ natural isomorphism between Hilbert spaces is an isomorphism defined without choosing bases of the spaces in question.

${ }^{20}$ Measurable in the sense that the inverse image of any Borel measurable subset of $M$ is Borel measurable in $M / N$.
} 
Lemma 3.3. The map $\chi$ in (35) is surjective.

Proof. Let $\sigma \in L^{2}(M / N, E / N)$. We will construct a section $s \in L_{c}^{2}(M, E)$ such that $\chi(s)=\sigma$, using the following diagram:

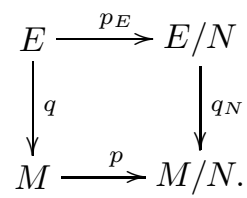

Here the horizontal maps are quotient maps and define principal fibre bundles, and the vertical maps are vector bundle projections.

Let $\left\{U_{j}\right\}$ be an open cover of $M / N$ that admits local trivialisations

$$
\begin{aligned}
& \tau_{j}: p^{-1}\left(U_{j}\right) \cong \\
& \theta_{j}^{N}: q_{N} \times N \\
& q_{j}\left(U_{j}\right) \stackrel{\cong}{\rightrightarrows} U_{j} \times E_{0} .
\end{aligned}
$$

Here $E_{0}$ is the typical fibre of $E$. Because $M / N$ is compact, the cover $\left\{U_{j}\right\}$ may be supposed to be finite. Via the isomorphism of vector bundles $p^{*}(E / N) \cong E$, the trivialisations $\theta_{j}^{N}$ induce local trivialisations of $E$ :

$$
\theta_{j}: q^{-1}\left(p^{-1}\left(U_{j}\right)\right) \stackrel{\cong}{\rightrightarrows} p^{-1}\left(U_{j}\right) \times E_{0} .
$$

And then, we can form trivialisations

$$
\tau_{j}^{E}: p_{E}^{-1}\left(q_{N}^{-1}\left(U_{j}\right)\right) \stackrel{\cong}{\rightrightarrows} q_{N}^{-1}\left(U_{j}\right) \times N,
$$

by

$$
\begin{aligned}
p_{E}^{-1}\left(q_{N}^{-1}\left(U_{j}\right)\right) & =q^{-1}\left(p^{-1}\left(U_{j}\right)\right) & & \\
& \cong p^{-1}\left(U_{j}\right) \times E_{0} & & \text { via } \theta_{j} \\
& \cong U_{j} \times N \times E_{0} & & \text { via } \tau_{j} \\
& \cong q_{N}^{-1}\left(U_{j}\right) \times N & & \text { via } \theta_{j}^{N} .
\end{aligned}
$$

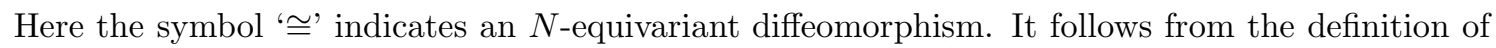
the trivialisation $\theta_{j}$ that $\tau_{j}^{E}$ composed with projection onto $q_{N}^{-1}\left(U_{j}\right)$ equals $p_{E}$, so that $\tau_{j}^{E}$ is indeed an isomorphism of principal $N$-bundles.

For every $j$, define the section $s_{j} \in L^{2}(M, E)$ by

$$
s_{j}\left(\tau_{j}^{-1}(\mathcal{O}, n)\right)=\left(\tau_{j}^{E}\right)^{-1}(\sigma(\mathcal{O}), n)
$$

for all $\mathcal{O} \in U_{j}$ and $n \in N$, and extended by zero outside $p^{-1}\left(U_{j}\right)$. By compactness ${ }^{21}$ of $M / N$, there is a compact subset $\widetilde{C} \subset M$ that intersects all $N$-orbits. Let $K \subset N$ be a compact subset of $d n$-volume 1 , and set $C:=K \cdot \widetilde{C}$. Then for all $m \in M$, the volume of the compact set

$$
V_{m}:=\left\{n \in N ; n^{-1} m \in C\right\}
$$

is at least 1. Define the section $\tilde{s}$ of $E$ by

$$
\tilde{s}(m)= \begin{cases}\sum_{j} s_{j}(m) & \text { if } m \in C \\ 0 & \text { if } m \notin C .\end{cases}
$$

\footnotetext{
${ }^{21}$ This is the only place where compactness of $M / N$ is used (the covering $\left\{U_{j}\right\}$ may also be locally finite). And even here, this assumption is not essential: it follows from this proof that all compactly supported $L^{2}$-sections of $E / N$ are in the image of $\chi$. Hence $\chi$ has dense image, so that the induced map from $L^{2}(M, E)_{N}$ to $L^{2}(M / N, E / N)$ is surjective. By small adaptations to the proofs of Propositions 3.2 and 3.8 everything in this section still applies if $M / N$ is noncompact. But because we assume that the quotient $M / \Gamma$ is compact anyway, we will take the lazy option and suppose that $M / N$ is compact.
} 
Then $\tilde{s} \in L_{c}^{2}(M, E)$, and for all $m \in M$,

$$
\begin{aligned}
\chi(\tilde{s})(N m) & =\sum_{\substack{j, N m \in U_{j}}} \int_{V_{m}} p_{E}\left(n \cdot s_{j}\left(n^{-1} m\right)\right) d n \\
& =\sum_{\substack{j, N m \in U_{j}}} \int_{V_{m}} p_{E}\left(\left(\tau_{j}^{E}\right)^{-1}\left(\sigma(N m), n \cdot \psi_{j}\left(n^{-1} m\right)\right)\right) d n,
\end{aligned}
$$

where $\left(N m, \psi_{j}\left(n^{-1} m\right)\right):=\tau_{j}\left(n^{-1} m\right)$. Now since $p_{E} \circ\left(\tau_{j}^{E}\right)^{-1}$ is projection onto $q_{N}^{-1}\left(U_{j}\right)$, the latter integral equals

$$
\#\left\{j ; N m \in U_{j}\right\} \operatorname{vol}\left(V_{m}\right) \sigma(N m) .
$$

Setting $\Phi(m):=\#\left\{j ; N m \in U_{j}\right\} \operatorname{vol}\left(V_{m}\right)$ gives a measurable function $\Phi$ on $M$ which is bounded below by 1 and $N$-invariant by invariance of $d n$. Hence

$$
s:=\frac{1}{\Phi} \tilde{s}
$$

is a section $s \in L_{c}^{2}(M, E)$ for which $\chi(s)=\sigma$.

\subsection{Differential operators}

Let $G$ and $E \rightarrow M$ be as in Section 3.2 Let $D: C^{\infty}(M, E) \rightarrow C^{\infty}(M, E)$ be a $G$-equivariant first order differential operator that is symmetric with respect to the $L^{2}$-inner product on compactly supported sections. Then $D$ defines an unbounded operator on $L^{2}(M, E)$. We assume that this operator has a self-adjoint extension, which we also denote by $D$.

\section{Functional calculus and properly supported operators}

Applying the functional calculus to the self-adjoint extension of $D$, we define the bounded, selfadjoint operator $b(D)$ on $L^{2}(M, E)$, for any bounded measurable function $b$ on $\mathbb{R}^{22}$ The operator $b(D)$ is $G$-equivariant because of the following result about functional calculus of unbounded operators, which follows directly from the definition as given for example in [58, page 261.

Lemma 3.4. Let $H$ be a Hilbert space, and let $\mathcal{D} \subset H$ be a dense subspace. Let $a: \mathcal{D} \rightarrow H$ be a self-adjoint operator. Let $H^{\prime}$ be another Hilbert space, and let $T: H \rightarrow H^{\prime}$ be a unitary isomorphism. Let $f$ be a measurable function on $\mathbb{R}$. Then

$$
T f(a) T^{-1}=f\left(T a T^{-1}\right) .
$$

We will later consider the case where $\left(L^{2}(M, E), b(D), \pi_{M}\right)$ is a $K$-homology cycle, and apply the map $V_{N}$ to this cycle. It is therefore important to us that the operator $b(D)$ is properly supported (Definition 3.1) for well-chosen functions $b$ :

Proposition 3.5. If $b$ is a bounded measurable function with compactly supported (distributional) Fourier transform $\hat{b}$, then the operator $b(D)$ is properly supported.

The proof of this proposition is based on the following two facts, whose proofs can be found in 31], Section 10.3.

Proposition 3.6. If $b$ is a bounded measurable function on $\mathbb{R}$ with compactly supported Fourier transform, then for all $s, t \in C_{c}^{\infty}(M, E)$,

$$
(b(D) s, t)_{L^{2}(M, E)}=\frac{1}{2 \pi} \int_{\mathbb{R}}\left(e^{i \lambda D} s, t\right)_{L^{2}(M, E)} \hat{b}(\lambda) d \lambda .
$$

\footnotetext{
${ }^{22}$ If $D$ is elliptic and $b$ is a normalising function, then $\left(L^{2}(M, E), b(D), \pi_{M}\right)$ is an equivariant $K$-homology cycle over $M$ (see Theorem 10.6.5 in [31).
} 
This is Proposition 10.3.5. from 31. By Stone's theorem, the operator $e^{i \lambda D}$ is characterised by the requirements that $\lambda \mapsto e^{i \lambda D}$ is a group homomorphism from $\mathbb{R}$ to the unitary operators on $L^{2}(M, E)$, and that for all $s \in C_{c}^{\infty}(M, E)$,

$$
\left.\frac{\partial}{\partial \lambda}\right|_{\lambda=0} e^{i \lambda D} s=i D s
$$

Lemma 3.7. Let $s \in C_{c}^{\infty}(M, E)$, and let $h \in C_{c}^{\infty}(M)$ be equal to 1 on the support of $s$. Let $\lambda \in \mathbb{R}$ such that $|\lambda|<\left\|\left[D, \pi_{M}(h)\right]\right\|^{-1}$. Then

$$
\operatorname{supp} e^{i \lambda D} s \subset \operatorname{supp} h .
$$

This follows from the proof of Proposition 10.3.1. from 31.

Proof of Proposition [3.5. Suppose supp $\hat{b} \subset[-R, R]$. Let $f \in C_{c}(M)$, and choose $h \in C_{c}^{\infty}(M)$ such that $h$ equals 1 on the support of $f$, and that $\left\|\left[D, \pi_{M}(h)\right]\right\| \leq \frac{1}{R}$. Let $1_{M}$ be the constant function 1 on $M$. Then by Lemma 3.7

$$
\pi_{M}\left(1_{M}-h\right) e^{i \lambda D} \pi_{M}(f)=0
$$

for all $\lambda \in]-R, R\left[\right.$. Here we have extended the nondegenerate representation $\pi_{M}$ of $C_{0}(M)$ on $L^{2}(M, E)$ to the multiplier algebra $C_{b}(M)$ of $C_{0}(M)$. So by Proposition 3.6 we have for all $s, t \in C_{c}^{\infty}(M, E)$,

$$
\begin{aligned}
\left(\pi_{M}\left(1_{M}-h\right) b(D) \pi_{M}(f) s, t\right)_{L^{2}(M, E)} & =\left(b(D) \pi_{M}(f) s, \pi_{M}\left(1_{M}-\bar{h}\right) t\right)_{L^{2}(M, E)} \\
& =\frac{1}{2 \pi} \int_{\mathbb{R}}\left(e^{i \lambda D} \pi_{M}(f) s, \pi_{M}\left(1_{M}-\bar{h}\right) t\right)_{L^{2}(M, E)} \hat{b}(\lambda) d \lambda \\
& =\frac{1}{2 \pi} \int_{-R}^{R}\left(\pi_{M}\left(1_{M}-h\right) e^{i \lambda D} \pi_{M}(f) s, t\right)_{L^{2}(M, E)} \hat{b}(\lambda) d \lambda \\
& =0,
\end{aligned}
$$

by (38). So

$$
\left(1-\pi_{M}(h)\right) b(D) \pi_{M}(f)=\pi_{M}\left(1_{M}-h\right) b(D) \pi_{M}(f)=0,
$$

and hence $b(D)$ is properly supported.

\section{The image of $b(D)$ under $V_{N}$}

Now suppose that $D$ is elliptic and that $b$ is a normalising function with compactly supported Fourier transform, ${ }^{23}$ so that $b(D)$ is the kind of operator that defines a $K$-homology class over $M$. Because $b(D)$ is properly supported, it preserves $L_{c}^{2}(M, E)$ and the construction used in the definition of the map $V_{N}$ applies to $b(D)$. The resulting operator $b(D)_{N}$ on $L^{2}(M, E)_{N}$ is defined by commutativity of the following diagram:

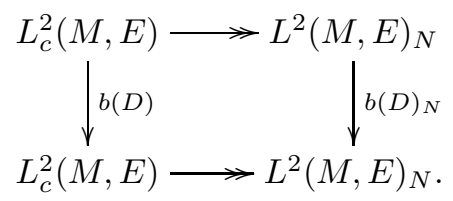

On the other hand, the operator $D$ induces an unbounded operator on $L^{2}(M / N, E / N)$, because it restricts to

$$
\tilde{D}^{N}: C^{\infty}(M, E)^{N} \rightarrow C^{\infty}(M, E)^{N},
$$

\footnotetext{
${ }^{23}$ If $g$ is a smooth, even, compactly supported function on $\mathbb{R}$, and $f:=g * g$ is its convolution square, then $b(\lambda):=\int_{\mathbb{R}} \frac{e^{i \lambda x}-1}{i x} f(x) d x$ is such a function (see [31], Exercise 10.9.3).
} 
from which we obtain

$$
D^{N}:=\psi_{E}^{-1} \tilde{D}^{N} \psi_{E}: C^{\infty}(M / N, E / N) \rightarrow C^{\infty}(M / N, E / N)
$$

(see Proposition 2.8). We regard $D^{N}$ as an unbounded operator on $L^{2}(M / N, E / N)$. It is symmetric with respect to the $L^{2}$-inner product, and hence essentially self-adjoint by 31, Corollary 10.2.6 We therefore have the bounded operator $b\left(D^{N}\right)$ on $L^{2}(M / N, E / N)$.

Our claim is:

Proposition 3.8. The isomorphism $\chi$ from Proposition 3.2 intertwines the operators $b(D)_{N}$ and $b\left(D^{N}\right)$ :

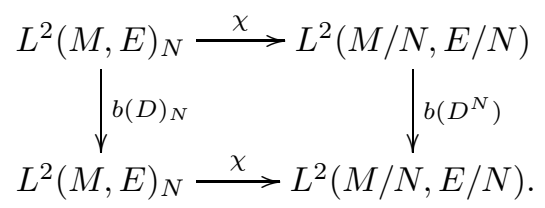

We will prove this claim by reducing it to the commutativity of another diagram. This diagram involves the Hilbert space $\tilde{L}^{2}(M / N, E / N)$, which is defined as the completion of the space $C^{\infty}(M, E)^{N}$ in the inner product

$$
(\sigma, \tau):=\int_{M / N}\left(\sigma(\varphi(\mathcal{O})), \tau(\varphi(\mathcal{O}))_{E} d \mathcal{O},\right.
$$

for any measurable section $\varphi: M / N \rightarrow M$. The map $\psi_{E}$ from Proposition 2.8 extends continuously to a unitary isomorphism

$$
\tilde{\psi}_{E}: \tilde{L}^{2}(M / N, E / N) \rightarrow L^{2}(M / N, E / N) .
$$

The unbounded operator $\tilde{D}^{N}$ on $\tilde{L}^{2}(M / N, E / N)$ is essentially self-adjoint because $D^{N}$ is, and because $\tilde{\psi}_{E}$ intertwines the two operators. Hence we have $b\left(\tilde{D}^{N}\right) \in \mathcal{B}\left(\tilde{L}^{2}(M / N, E / N)\right)$. We will deduce Proposition 3.8 from

Lemma 3.9. The following diagram commutes:

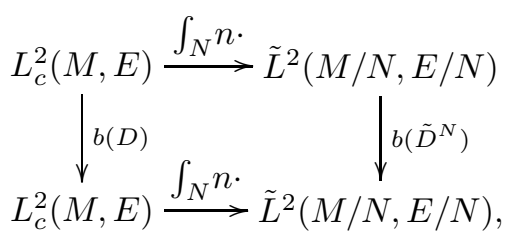

where the map $\int_{N} n \cdot$ is given by ${ }^{24}$

$$
\left(\int_{N} n \cdot(s)\right)(N m)=\int_{N} n \cdot s\left(n^{-1} m\right) d n .
$$

Proof. Step 1. Because the representation of $N$ in $L^{2}(M, E)$ is unitary, we have

$$
\left(\int_{N} n \cdot(s), t\right)_{L^{2}(M, E)}=\left(s, \int_{N} n \cdot(t)\right)_{L^{2}(M, E)}
$$

for all $s, t \in L_{c}^{2}(M, E)$.

Step 2. By Proposition 3.10 below and equivariance of $D$, we have

$$
\left(\int_{N} n \cdot\right) \circ D=\tilde{D}^{N} \circ \int_{N} n
$$

\footnotetext{
${ }^{24}$ Note that the space $\tilde{L}^{2}(M / N, E / N)$ can be realised as a space of sections of $E$.
} 
on $C_{c}^{\infty}(M, E)$.

Step 3. For all $s \in C_{c}^{\infty}(M, E)$, we have by Proposition 3.10

$$
\begin{aligned}
\left.\frac{\partial}{\partial \lambda}\right|_{\lambda=0} \int_{N} n \cdot \circ e^{i \lambda D} s & =\int_{N} \frac{\partial}{\partial \lambda} e^{i \lambda D} n \cdot s d n \\
& =i \int_{N} n \cdot D s d n \\
& =i \tilde{D}^{N} \int_{N} n \cdot(s) \quad \text { (by Step 2) } \\
& =\left.\frac{\partial}{\partial \lambda}\right|_{\lambda=0} e^{i \lambda \tilde{D}^{N}} \int_{N} n \cdot(s) .
\end{aligned}
$$

So by Stone's theorem,

for all $\lambda \in \mathbb{R}$.

$$
\int_{N} n \cdot \circ e^{i \lambda D}=e^{i \lambda \tilde{D}^{N}} \circ \int_{N} n
$$

Step 4. For all $s, t \in C_{c}^{\infty}(M, E)$,

$$
\begin{array}{rlrl}
\left(b\left(\tilde{D}^{N}\right) \int_{N} n \cdot(s), t\right)_{L^{2}(M, E)} & =\frac{1}{2 \pi} \int_{\mathbb{R}}\left(e^{i \lambda \tilde{D}^{N}} \int_{N} n \cdot(s), t\right)_{L^{2}(M, E)} \hat{b}(\lambda) d \lambda & \text { (by Proposition 3.6) } \\
& =\frac{1}{2 \pi} \int_{\mathbb{R}}\left(\int_{N} n \cdot e^{i \lambda D} s, t\right)_{L^{2}(M, E)} \hat{b}(\lambda) d \lambda & \text { (by Step 3) } \\
& =\frac{1}{2 \pi} \int_{\mathbb{R}}\left(e^{i \lambda D} s, \int_{N} n \cdot(t)\right)_{L^{2}(M, E)} \hat{b}(\lambda) d \lambda & \text { (by Step 1) } \\
& =\left(b(\tilde{D}) s, \int_{N} n \cdot(t)\right)_{L^{2}(M, E)} \quad \text { (by Proposition 3.6) } \\
& =\left(\int_{N} n \cdot b(\tilde{D}) s, t\right)_{L^{2}(M, E)} \quad \text { (by Step 1). }
\end{array}
$$

This completes the proof.

Proposition 3.10. Let $M_{1}$ and $M_{2}$ be manifolds, and suppose $M_{2}$ is equipped with a measure $d m_{2}$. Let $E \rightarrow M_{1}$ be a vector bundle, and let

$$
D: C^{\infty}\left(M_{1}, E\right) \rightarrow C^{\infty}\left(M_{1}, E\right)
$$

be a differential operator. Let $p_{1}: M_{1} \times M_{2} \rightarrow M_{1}$ be projection onto the first factor, and consider the operator

$$
p_{1}^{*} D: C^{\infty}\left(M_{1} \times M_{2}, p_{1}^{*} E\right) \rightarrow C^{\infty}\left(M_{1} \times M_{2}, p_{1}^{*} E\right),
$$

defined by $\left(p_{1}^{*} D\right) p_{1}^{*} \sigma=p_{1}^{*}(D \sigma)$ for all $\sigma \in C^{\infty}\left(M_{1}, E\right)$.

Then for all $s \in C^{\infty}\left(M_{1} \times M_{2}, p_{1}^{*} E\right)$ and all $m_{1} \in M_{1}$,

$$
D\left(\int_{M_{2}} s\left(\cdot, m_{2}\right) d m_{2}\right)\left(m_{1}\right)=\int_{M_{2}} p_{1}^{*} D s\left(m_{1}, m_{2}\right) d m_{2} .
$$

We now derive Proposition 3.8 from Lemma 3.9 Proof of Proposition 3.8. Consider the following cube:

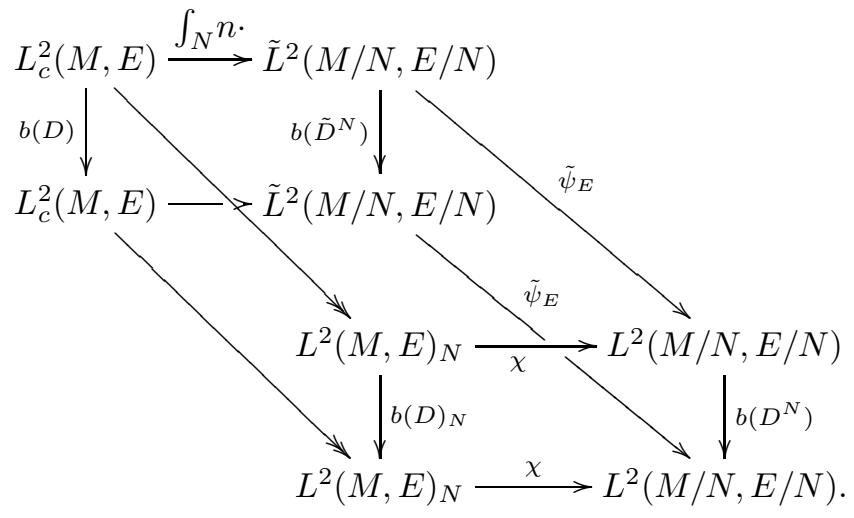


The rear square (with the operators $b(D)$ and $b\left(\tilde{D}^{N}\right)$ in it) commutes by Lemma 3.9 The left hand square (with the operators $b(D)$ and $\left.b(D)_{N}\right)$ commutes by definition of $b(D)_{N}$, and the right hand square (with $b\left(\tilde{D}^{N}\right)$ and $b\left(D^{N}\right)$ ) commutes by Lemma 3.4 The top and bottom squares commute by definition of the map $\chi$, so that the front square commutes as well, which is Proposition 3.8 .

\subsection{Multiplication of sections by functions}

Let $G, M$ and $E$ be as in Subsections 3.2 and 3.3 As before, let

$$
\pi_{M}: C_{0}(M) \rightarrow \mathcal{B}\left(L^{2}(M, E)\right)
$$

and

$$
\pi_{M / N}: C_{0}(M / N) \rightarrow \mathcal{B}\left(L^{2}(M / N, E / N)\right)
$$

be the representations defined by multiplication of sections by functions. Let

$$
\left(\pi_{M}\right)_{N}: C_{0}(M / N) \rightarrow \mathcal{B}\left(L^{2}(M, E)_{N}\right)
$$

be the representation obtained from $\pi_{M}$ by the procedure in Subsection 3.1

Lemma 3.11. The isomorphism (36) intertwines the representations $\left(\pi_{M}\right)_{N}$ and $\pi_{M / N}$.

Proof. The representation $\left(\pi_{M}\right)_{N}$ is induced by

$$
\begin{aligned}
& \pi_{M}^{N}: C(M / N) \rightarrow \mathcal{B}\left(L_{c}^{2}(M, E)\right), \\
& \left(\pi_{M}^{N}(f) s\right)(m)=f(N \cdot m) s(m) .
\end{aligned}
$$

For all $f \in C(M / N), s \in L_{c}^{2}(M, E)$ and $m \in M$, we have

$$
\begin{aligned}
\chi\left(\pi_{M}^{N}(f) s\right)(N \cdot m) & =N \cdot \int_{N} n \cdot f\left(N \cdot n^{-1} m\right) s\left(n^{-1} \cdot m\right) d n \\
& =N \cdot f(N \cdot m) \int_{N} n \cdot s\left(n^{-1} \cdot m\right) d n \\
& =\left(\pi_{M / N}(f) \chi(s)\right)(N \cdot m) .
\end{aligned}
$$

\subsection{Conclusion}

Let $G, M, E, D, D^{N}, \pi_{M}$ and $\pi_{M / N}$ be as in Sections 3.2 - 3.4 Suppose that the vector bundle $E$ carries a $\mathbb{Z}_{2}$-grading with respect to which the operator $D$ is odd. Suppose $D$ is elliptic and essentially self-adjoint as an unbounded operator on $L^{2}(M, E) .{ }^{25}$. Let $b$ be a normalising function with compactly supported Fourier transform. Then Proposition 3.2 Proposition 3.8 and Lemma 3.11 may be summarised as follows.

Theorem 3.12. Let $\left(L^{2}(M, E)_{N}, b(D)_{N},\left(\pi_{M}\right)_{N}\right)$ be the triple obtained from $\left(L^{2}(M, E), b(D), \pi_{M}\right)$ by the procedure of Subsection 3.1. Then there is a unitary isomorphism

$$
\chi: L^{2}(M, E)_{N} \rightarrow L^{2}(M / N, E / N)
$$

that intertwines the representations of $G / N$, the operators $b(D)_{N}$ and $b\left(D^{N}\right)$, and the representations $\left(\pi_{M}\right)_{N}$ and $\pi_{M / N}$.

Corollary 3.13. The image of the class

$$
[D]:=\left[L^{2}(M, E), b(D), \pi_{M}\right] \in K_{0}^{G}(M)
$$

under the homomorphism $V_{N}$ defined in Subsection 3.1 is

$$
V_{N}[D]=\left[L^{2}(M / N, E / N), b\left(D^{N}\right), \pi_{M / N}\right]=:\left[D^{N}\right] \in K_{0}^{G / N}(M / N) .
$$

\footnotetext{
${ }^{25}$ This is the case if $M$ is complete and $D$ is a Dirac operator on $M$, see footnote 16
} 
Remark 3.14. If the action of $G$ on $M$ happens to be free, then Corollary 3.13 allows us to restate the Guillemin-Sternberg conjecture 1.1 without using techniques from noncommutative geometry. Indeed, for free actions we have

$$
\begin{aligned}
R_{Q} \circ \mu_{M}^{G}\left[\not D_{M}^{L}\right] & =\mu_{M / G}^{\{e\}} \circ V_{G}\left[\not D_{M}^{L}\right] \quad \text { by naturality of } \mu \\
& =\operatorname{index}\left(\not D_{M}^{L}\right)^{G} \quad \text { by Corollary } 3.13 \\
& =\operatorname{dim}\left(\operatorname{ker}\left(\not D_{M}^{L}\right)^{+}\right)^{G}-\operatorname{dim}\left(\operatorname{ker}\left(\not D_{M}^{L}\right)^{-}\right)^{G} \in \mathbb{Z}
\end{aligned}
$$

Here the kernels of $\left(\not D_{M}^{L}\right)^{ \pm}$are taken in the spaces of smooth, not necessarily $L^{2}$, sections of $\bigwedge^{0, *} T^{*} M \otimes L$. Note that even though these kernels may be infinite-dimensional, their $G$-invariant parts are not, because they are the kernels of the elliptic operators $\left(\left(\not D_{M}^{L}\right)^{ \pm}\right)^{G}$ on the compact manifold $M / G$. So Conjecture 1.1 becomes

$$
\operatorname{index} \not D_{M_{G}}^{L_{G}}=\operatorname{dim}\left(\operatorname{ker}\left(\not D_{M}^{L}\right)^{+}\right)^{G}-\operatorname{dim}\left(\operatorname{ker}\left(\not D_{M}^{L}\right)^{-}\right)^{G} .
$$

Unfortunately, in our situation this argument would only apply to discrete groups (see Remark [2.2).

\section{Dirac operators}

In this section, we make the assumptions stated in Subsection 2.1] In particular, $\Gamma$ is a normal discrete subgroup of $G$. The goal of this section is to prove that Theorem 2.9 is a special case of Corollary 3.13

Proposition 4.1. Consider the Dolbeault-Dirac operator $\not D_{M}^{L}$ on $\Omega^{0, *}(M ; L)$, and the induced operator $\left(\not D_{M}^{L}\right)^{\Gamma}$ on $C^{\infty}\left(M / \Gamma,\left(\bigwedge^{0, *} T^{*} M \otimes L\right) / \Gamma\right)$. There is an isomorphism

$$
\Xi: \Omega^{0, *}(M / \Gamma ; L / \Gamma) \rightarrow C^{\infty}\left(M / \Gamma,\left(\bigwedge^{0, *} T^{*} M \otimes L\right) / \Gamma\right)
$$

that is isometric with respect to the $L^{2}$-inner product and intertwines the Dolbeault-Dirac operator $\not D_{M / \Gamma}^{L / \Gamma}$ on $\Omega^{0, *}(M / \Gamma ; L / \Gamma)$ and the operator $\left(\not D_{M}^{L}\right)^{\Gamma}$.

Consequently, $\Xi$ induces a unitary isomorphism between the corresponding $L^{2}$-spaces, which by Lemma 3.4 intertwines the bounded operators obtained from $\not D_{M / \Gamma}^{L / \Gamma}$ and $\left(\not D_{M}^{L}\right)^{\Gamma}$ using a normalising function with compactly supported Fourier transform. Hence Theorem 2.9 follows, as

$$
\begin{array}{rlr}
V_{\Gamma}\left(\left[\not D_{M}^{L}\right]\right) & =\left[\left(\not D_{M}^{L}\right)^{\Gamma}\right] \quad \text { by Corollary } 3.13 \\
& =\left[\not D_{M / \Gamma}^{L / \Gamma}\right] \quad \text { by Proposition 4.1 }
\end{array}
$$

\subsection{The isomorphism}

The isomorphism of $C^{\infty}(M / \Gamma)$-modules $\Xi$ in Proposition 4.1 is defined as follows. The quotient map $p: M \rightarrow M / \Gamma$ induces the vector bundle homomorphism

$$
\bigwedge T p^{*}: \wedge T^{*}(M / \Gamma) \rightarrow \wedge T^{*} M
$$

Because $T p$ intertwines the almost complex structures on $T M$ and $T(M / \Gamma)$, the homomorphism (39) induces

$$
\bigwedge^{0, *} T p^{*}: \bigwedge^{0, *} T^{*}(M / \Gamma) \rightarrow \bigwedge^{0, *} T^{*} M
$$


Composition with the quotient map $T^{*} M \rightarrow\left(T^{*} M\right) / \Gamma$ turns (39) and (40) into isomorphisms

$$
\begin{aligned}
& \left(\bigwedge T p^{*}\right)^{\Gamma}: \bigwedge T^{*}(M / \Gamma) \rightarrow\left(\bigwedge T^{*} M\right) / \Gamma ; \\
& \left(\bigwedge^{0, *} T p^{*}\right)^{\Gamma}: \bigwedge^{0, *} T^{*}(M / \Gamma) \rightarrow\left(\bigwedge^{0, *} T^{*} M\right) / \Gamma .
\end{aligned}
$$

On the spaces of smooth sections of the vector bundles in question, the isomorphisms (41) and (42) induce isomorphisms of $C^{\infty}(M / \Gamma)$-modules

$$
\begin{gathered}
\Psi: \Omega^{*}(M / \Gamma) \rightarrow C^{\infty}\left(M / \Gamma,\left(\bigwedge T^{*} M\right) / \Gamma\right) \\
\Psi^{0, *}: \Omega^{0, *}(M / \Gamma) \rightarrow C^{\infty}\left(M / \Gamma,\left(\bigwedge^{0, *} T^{*} M\right) / \Gamma\right) .
\end{gathered}
$$

Now the isomorphism $\Xi$ is defined as

$$
\begin{aligned}
& \Xi: \Omega^{0, *}(M / \Gamma ; L / \Gamma) \cong \\
& \Omega^{0, *}(M / \Gamma) \otimes_{C^{\infty}(M / \Gamma)} C^{\infty}(M / \Gamma, L / \Gamma) \stackrel{\Psi^{0, *} \otimes 1_{C^{\infty}(M / \Gamma, L / \Gamma)}}{\longrightarrow} \\
& C^{\infty}\left(M / \Gamma,\left(\bigwedge^{0, *} T^{*} M\right) / \Gamma\right) \otimes_{C^{\infty}(M / \Gamma)} C^{\infty}(M / \Gamma, L / \Gamma) \\
& \cong C^{\infty}\left(M / \Gamma,\left(\bigwedge^{0, *} T^{*} M \otimes L\right) / \Gamma\right) .
\end{aligned}
$$

It is isometric by definition of the measure $d \mathcal{O}$ on $M / \Gamma$ and the metrics on the vector bundles involved. Therefore, it remains to prove that $\Xi$ intertwines the operators $\not D_{M / \Gamma}^{L / \Gamma}$ and $\left(\not D_{M}^{L}\right)^{\Gamma}$.

\subsection{Proof of Proposition 4.1}

\section{The connections}

Recall the isomorphism of $C^{\infty}(M)^{\Gamma} \cong C^{\infty}(M / \Gamma)$-modules $\psi_{E}: C^{\infty}(M, E)^{\Gamma} \rightarrow C^{\infty}(M / \Gamma, E / \Gamma)$ defined by (31), with $H=\Gamma$, for any $\Gamma$-vector bundle $E$ over $M$. Also consider the pullback $p^{*}$ of differential forms on $M / \Gamma$ to invariant differential forms on $M$. It defines an isomorphism of $C^{\infty}(M / \Gamma) \cong C^{\infty}(M)^{\Gamma}$-modules

$$
p^{*}: \Omega^{*}(M / \Gamma) \rightarrow \Omega^{*}(M)^{\Gamma}
$$

Lemma 4.2. The following diagram commutes:

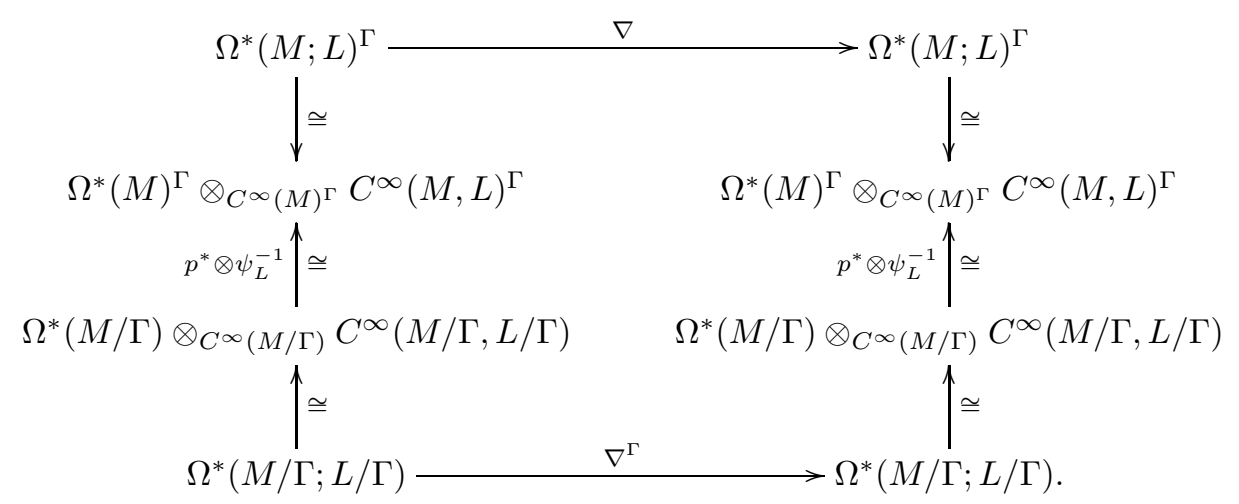

The proof of this lemma is a matter of writing out definitions.

By definition of the almost complex structure on $T(M / \Gamma)$, we have

$$
p^{*}\left(\Omega^{0, q}(M / \Gamma)\right)=\Omega^{0, q}(M)^{\Gamma}
$$


for all $q$. Therefore, Lemma 4.2 implies that the following diagram commutes:

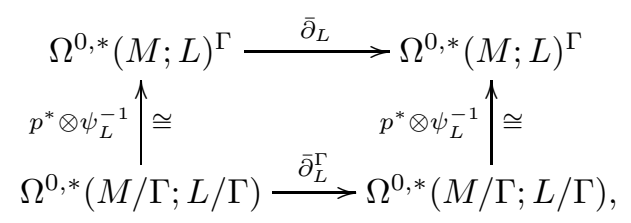

with $\bar{\partial}_{L}$ and $\bar{\partial}_{L}^{\Gamma}$ as in Subsection 2.2

\section{The Dirac operators}

By definition of the measure $d \mathcal{O}$ on $M / \Gamma$ and the metrics $B^{\Gamma}$ on $T(M / \Gamma)$ and $H^{\Gamma}$ on $L / \Gamma$, the isomorphism

$$
p^{*} \otimes \psi_{L}^{-1}: \Omega^{0, *}(M / \Gamma ; L / \Gamma) \rightarrow \Omega^{0, *}(M ; L)^{\Gamma}
$$

is isometric with respect to the inner product on $\Omega^{0, *}(M / \Gamma ; L / \Gamma)$ defined by

$$
(\alpha \otimes \sigma, \beta \otimes \tau)=\int_{M / \Gamma} B^{\Gamma}(\alpha, \beta) H^{\Gamma}(\sigma, \tau) d \mathcal{O},
$$

for all $\alpha, \beta \in \Omega^{0, *}(M / \Gamma)$ and $\sigma, \tau \in C^{\infty}(M / \Gamma, L / \Gamma)$, and the inner product on $\Omega^{0, *}(M ; L)^{\Gamma}$ defined by

$$
(\zeta \otimes s, \xi \otimes t)=\int_{U} B(\zeta, \xi) H(s, t) d m,
$$

for all $\zeta, \xi \in \Omega^{0, *}(M)^{\Gamma}$ and $s, t \in C^{\infty}(M, L)^{\Gamma}$. (Recall that $U \subset M$ is a fundamental domain for the $\Gamma$-action.)

The Dolbeault-Dirac operators on $M$ and $M / \Gamma$ are defined by

$$
\begin{gathered}
\not D_{M}^{L}=\bar{\partial}_{L}+\bar{\partial}_{L}^{*} ; \\
\not D_{M / \Gamma}^{L / \Gamma}=\bar{\partial}_{L}^{\Gamma}+\left(\bar{\partial}_{L}^{\Gamma}\right)^{*} .
\end{gathered}
$$

Here the formal adjoint $\left(\bar{\partial}_{L}^{\Gamma}\right)^{*}$ is defined with respect to the inner product (46). The formal adjoint $\bar{\partial}_{L}^{*}$ is defined by

$$
\int_{M}(B \otimes H)\left(\bar{\partial}_{L}^{*} \eta, \theta\right) d m=\int_{M}(B \otimes H)\left(\eta, \bar{\partial}_{L} \theta\right) d m,
$$

for all $\eta, \theta \in \Omega^{0, *}(M ; L), \theta$ with compact support. But this is actually the same as the formal adjoint of $\bar{\partial}_{L}$ with respect to the inner product [47):

Lemma 4.3. Let $\Gamma$ be a discrete group, acting properly and freely on a manifold $M$, equipped with $a \Gamma$-invariant measure $d m$. Suppose $M / \Gamma$ is compact. Let $E \rightarrow M$ be a $\Gamma$-vector bundle, equipped with a $\Gamma$-invariant metric $\langle\cdot, \cdot\rangle$. Let

$$
D: C^{\infty}(M, E) \rightarrow C^{\infty}(M, E)
$$

be a $\Gamma$-equivariant differential operator. Let

$$
D^{*}: C^{\infty}(M, E) \rightarrow C^{\infty}(M, E)
$$

be the operator such that for all $s, t \in C^{\infty}(M, E), t$ with compact support,

$$
\int_{M}\left\langle D^{*} s, t\right\rangle d m=\int_{M}\langle s, D t\rangle d m .
$$

Let $U \subset M$ be a fundamental domain for the $\Gamma$-action. Then the restriction of $D^{*}$ to $C^{\infty}(M, E)^{\Gamma}$ satisfies

$$
\int_{U}\left\langle D^{*} s, t\right\rangle d m=\int_{U}\langle s, D t\rangle d m,
$$

for all $s, t \in C^{\infty}(M, E)^{\Gamma}$. 
Proof. We will show that for all $s \in C^{\infty}(M, E)^{\Gamma}$, and all $t$ in a dense subspace of $C^{\infty}(M, E)^{\Gamma}$, we have

$$
\int_{U}\left\langle D^{*} s, t\right\rangle d m=\int_{U}\langle s, D t\rangle d m .
$$

Let $\tau$ be a section of $E$, with compact support in $U$. Define the section $t$ of $E$ by extending the restriction $\left.\tau\right|_{U} \Gamma$-invariantly to $M$. The space of all sections $t$ obtained in this way is dense in $C^{\infty}(M, E)^{\Gamma}$ with respect to the topology induced by the inner product

$$
(s, t):=\int_{U}\langle s, t\rangle d m
$$

Then for all $s \in C^{\infty}(M, E)^{\Gamma}$,

$$
\int_{U}\left\langle D^{*} s, t\right\rangle d m=\int_{M}\left\langle D^{*} s, \tau\right\rangle d m=\int_{M}\langle s, D \tau\rangle d m=\int_{U}\langle s, D t\rangle d m .
$$

We conclude that $p^{*} \otimes \psi_{L}^{-1}$ is an isometric isomorphism with respect to the inner products used to define the adjoints $\bar{\partial}_{L}^{*}$ and $\left(\bar{\partial}_{L}^{\Gamma}\right)^{*}$. Hence the commutativity of diagram (45) implies

Corollary 4.4. The following diagram commutes:

$$
\begin{array}{cc}
\Omega^{0, *}(M ; L)^{\Gamma} \stackrel{D_{M}^{L}}{\longrightarrow} \Omega^{0, *}(M ; L)^{\Gamma} \\
p^{*} \otimes \psi_{L}^{-1} \uparrow \cong \quad \\
\Omega^{0, *}(M / \Gamma ; L / \Gamma) \stackrel{\not_{M / \Gamma}^{L / \Gamma}}{\longrightarrow} \Omega^{*} \otimes \psi_{L}^{-1} \uparrow \cong \\
& \Omega^{0, *}(M / \Gamma ; L / \Gamma) .
\end{array}
$$

Remark 4.5. Corollary 4.4 shows that for discrete groups a much stronger statement than the Guillemin-Sternberg conjecture holds. Indeed, by Remark 3.14 the Guillemin-Sternberg conjecture states that the restriction of the operator $\not_{M}^{L}$ to $\Omega^{0, *}(M ; L)^{\Gamma}$ is related to the operator $\not_{M / \Gamma}^{L / \Gamma}$ by the fact that their indices are equal (as operators on smooth, not necessarily $L^{2}$, sections). But these operators are in fact more strongly related: they are intertwined by an isometric isomorphism.

\section{End of the proof of Proposition 4.1}

The last step in the proof of Proposition 4.1 is a decomposition of the isomorphism

$$
p^{*}: \Omega^{*}(M / \Gamma) \rightarrow \Omega^{*}(M)^{\Gamma} .
$$

Lemma 4.6. The following diagram commutes:

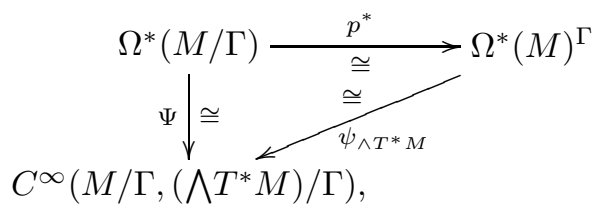

where $\Psi$ is the isomorphism (43).

The proof of this lemma is a short and straightforward computation.

Proof of Proposition [4.1. Together with Lemma 4.6 and the definition of the operator

$$
\left(\not D_{M}^{L}\right)^{\Gamma}: C^{\infty}\left(M / \Gamma,\left(\bigwedge^{0, *} T^{*} M \otimes L\right) / \Gamma\right) \rightarrow C^{\infty}\left(M / \Gamma,\left(\bigwedge^{0, *} T^{*} M \otimes L\right) / \Gamma\right),
$$


Corollary 4.4 implies that the following diagram commutes:

$$
\begin{aligned}
& \Omega^{0, *}(M ; L)^{\Gamma} \longrightarrow \mathbb{D}_{M}^{L} \longrightarrow \Omega^{0, *}(M ; L)^{\Gamma} \\
& \psi_{\wedge 0, * T^{*} M} \otimes \psi_{L} \mid \cong \quad \psi_{\wedge 0, *_{T}{ }^{*} M} \otimes \psi_{L} \downarrow \cong \\
& C^{\infty}\left(M / \Gamma,\left(\bigwedge^{0, *} T^{*} M \otimes L\right) / \Gamma\right) \stackrel{\left(D_{M}^{L}\right)^{\Gamma}}{\longrightarrow} C^{\infty}\left(M / \Gamma,\left(\bigwedge^{0, *} T^{*} M \otimes L\right) / \Gamma\right)
\end{aligned}
$$

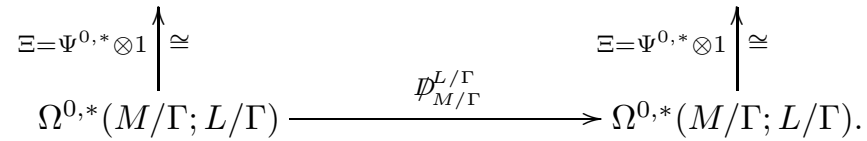

Indeed, the outside diagram commutes by Corollary 4.4 and Lemma 4.6] and the upper square commutes by definition of $\left(\not D_{M}^{L}\right)^{\Gamma}$. Hence the lower square commutes as well, which is Proposition 4.1

\section{$5 \quad$ Abelian discrete groups}

In this section, we consider the situation of Section 2 with the additional assumption that $G=\Gamma$ is an abelian discrete group. Then the Guillemin-Sternberg conjecture can be proved directly, without using naturality of the assembly map (33). This proof is based on Proposition 4.1 and the description of the assembly map in this special case given by Baum, Connes and Higson [5], Example 3.11 (which in turn is based on Lusztig [44). We will first explain this example in a little more detail than given in [5], and then show how it implies Theorem [1.2 for abelian discrete groups.

\subsection{The assembly map for abelian discrete groups}

The proof of the Guillemin-Sternberg conjecture for discrete abelian groups is based on the following result:

Proposition 5.1. Let $M, E, D$ and $D^{\Gamma}$ be as in Subsection 3.5. Suppose that $G=\Gamma$ is abelian and discrete. Using the normalising function $b(x)=\frac{x}{\sqrt{1+x^{2}}}$, we form the operator $F:=b(D)$, so that we have the class

$$
\left[L^{2}(M, E), F\right] \in K_{0}^{\Gamma}(M) .
$$

Then ${ }^{26}$

$$
R_{Q}^{(\Gamma)} \circ \mu_{M}^{\Gamma}\left[L^{2}(M, E), F\right]=\operatorname{index} D^{\Gamma} .
$$

In view of Proposition 4.1] Proposition 5.1 implies our Guillemin-Sternberg conjecture (i.e. Theorem 1.2) for discrete abelian groups.

\section{Kernels of operators as vector bundles}

Using Example 3.11 from [5], we can explicitly compute

$$
\left[\mathcal{E}, F_{\mathcal{E}}\right]:=\mu_{M}^{\Gamma}\left[L^{2}(M, E), F\right] \in K K_{0}\left(\mathbb{C}, C^{*}(\Gamma)\right) .
$$

Note that since $\Gamma$ is discrete, its unitary dual $\hat{\Gamma}$ is compact. And because $\Gamma$ is abelian, all irreducible unitary representations are of the form

$$
U_{\alpha}: \Gamma \rightarrow U(1)
$$

for $\alpha \in \hat{\Gamma}$. Fourier transform defines an isomorphism $C^{*}(\Gamma) \cong C_{0}(\hat{\Gamma})$. Therefore,

$$
K K_{0}\left(\mathbb{C}, C^{*}(\Gamma)\right) \cong K_{0}\left(C^{*}(\Gamma)\right) \cong K_{0}\left(C_{0}(\hat{\Gamma})\right) \cong K^{0}(\hat{\Gamma}) .
$$

\footnotetext{
${ }^{26}$ Recall that we abuse notation by writing index $D^{\Gamma}:=\operatorname{dim} \operatorname{ker}\left(D^{\Gamma}\right)^{+}-\operatorname{dim} \operatorname{ker}\left(D^{\Gamma}\right)^{-}$.
} 
Because $\hat{\Gamma}$ is compact, the image of $\left[\mathcal{E}, F_{\mathcal{E}}\right]$ in $K^{0}(\hat{\Gamma})$ is the difference of the isomorphism classes of two vector bundles over $\hat{\Gamma}$. These two vector bundles can be determined as follows. For all $\alpha \in \hat{\Gamma}$, we define the Hilbert space $H_{\alpha}$ as the space of all measurable sections $s_{\alpha}$ of $E$ (modulo equality almost everywhere), such that for all $\gamma \in \Gamma$,

$$
\gamma \cdot s_{\alpha}=U_{\alpha}(\gamma)^{-1} s_{\alpha}
$$

and such that the norm

$$
\left\|s_{\alpha}\right\|_{\alpha}^{2}=\left\langle s_{\alpha}, s_{\alpha}\right\rangle_{\alpha}
$$

is finite, where the inner product $\langle\cdot, \cdot\rangle_{\alpha}$ is defined by

$$
\left\langle s_{\alpha}, t_{\alpha}\right\rangle_{\alpha}:=\int_{M / \Gamma}\left(s_{\alpha}(\varphi(\mathcal{O})), t_{\alpha}(\varphi(\mathcal{O}))\right)_{E} d \mathcal{O},
$$

where $\varphi$ is any measurable section of the principal fibre bundle $M \rightarrow M / \Gamma$. The space $H_{\alpha}$ is isomorphic to the space of $L^{2}$-sections of the vector bundle $E_{\alpha}$, where

$$
E_{\alpha}:=E /\left(\gamma \cdot e \sim U_{\alpha}^{-1}(\gamma) e\right) \rightarrow M / \Gamma .
$$

Let $H_{\alpha}^{D}$ be the dense subspace

$$
H_{\alpha}^{D}:=\left\{s_{\alpha} \in H_{\alpha} \cap C^{\infty}(M, E) ; D s_{\alpha} \in H_{\alpha}\right\} \subset H_{\alpha} .
$$

Because the operator $D$ is $\Gamma$-equivariant, it restricts to an unbounded operator

$$
D_{\alpha}: H_{\alpha}^{D} \rightarrow H_{\alpha}
$$

on $H_{\alpha}$. It is essentially self-adjoint by [31, Corollary 10.2.6., and hence induces the bounded operator

$$
F_{\alpha}:=\frac{D_{\alpha}}{\sqrt{1+D_{\alpha}^{2}}} \in \mathcal{B}\left(H_{\alpha}\right) .
$$

The grading on $E$ induces a grading on $H_{\alpha}$ with respect to which $D_{\alpha}$ and $F_{\alpha}$ are odd. The operators $F_{\alpha}$ are elliptic pseudo-differential operators:

Lemma 5.2. Let $D$ be an elliptic, first order differential operator on a vector bundle $E \rightarrow M$, and suppose $D$ defines an essentially self-adjoint operator on $L^{2}(M, E)$ with respect to some measure on $M$ and metric on $E$. Then the operator $F:=\frac{D}{\sqrt{1+D^{2}}}$ is an elliptic pseudo-differential operator.

Proof. This result seems well known and is easily derived from a number of related results in the literature. It is, in any case, sufficient to show that $\left(1+D^{2}\right)^{-\frac{1}{2}}$ is a pseudo- differential operator. The following proof was communicated to us by Elmar Schrohe. ${ }^{27}$

According to [6], a bounded operator $A: L^{2}\left(\mathbb{R}^{n}\right) \rightarrow L^{2}\left(\mathbb{R}^{n}\right)$ is a pseudo-differential operator on $\mathbb{R}^{n}$ iff all iterated commutators with $x_{j}$ (as a multiplication operator) and $\partial_{x_{j}}$ are bounded operators. This immediately yields the lemma for $M=\mathbb{R}^{n}$ (cf. [6], Theorem 4.2). To extend this result to the manifold case, we recall that an operator $A: C^{\infty}(M) \rightarrow \mathcal{D}^{\prime}(M)$ on a manifold $M$ is a pseudo-differential operator when for each choice of smooth functions $f, g$ with support in a single coordinate neighbourhood, $f A g$ is a pseudo-differential operator on $\mathbb{R}^{n}$. (Here one has to admit nonconnected coordinate neighbourhoods.)

Now write $\left(1+D^{2}\right)^{-\frac{1}{2}}$ as a Dunford integral (cf. [17, pp. 556-577), as follows:

$$
\left(1+D^{2}\right)^{-\frac{1}{2}}=\oint_{C} \frac{d z}{2 \pi i}(1+z)^{-\frac{1}{2}}\left(z-D^{2}\right)^{-1}
$$

\footnotetext{
${ }^{27}$ An independent proof was suggested to us by John Roe, who mentioned that in the case at hand the functional calculus for (pseudo) differential operators developed in 68 for compact manifolds may be extended to the noncompact case. A third proof may be constructed using heat kernel techniques, as in the unpublished Diplomarbeit of Hanno Sahlmann (Rainer Verch, private communication).
} 
To compute the commutators of $f\left(1+D^{2}\right)^{-\frac{1}{2}} g$ with $x_{j}$ and $\partial_{x_{j}}$, one may take these inside the contour integral. Boundedness of all iterated commutators then easily follows, using the fact that $f$ and $g$ have compact support.

The same argument, with the exponent $-\frac{1}{2}$ replaced by $\frac{1}{2}$, shows that $\left(1+D^{2}\right)^{\frac{1}{2}}$ is a pseudodifferential operator, and ellipticity of $\left(1+D^{2}\right)^{-\frac{1}{2}}$ follows.

Consider the field of Hilbert spaces

$$
\left(H_{\alpha}\right)_{\alpha \in \hat{\Gamma}} \rightarrow \hat{\Gamma}
$$

In the next subsection, we will give this field the structure of a continuous field of Hilbert spaces by specifying its space of continuous sections $C\left(\hat{\Gamma},\left(H_{\alpha}\right)_{\alpha \in \hat{\Gamma}}\right)$. Consider the subfields

$$
\begin{aligned}
& \left(\operatorname{ker} D_{\alpha}^{+}\right)_{\alpha \in \hat{\Gamma}} \rightarrow \hat{\Gamma} \\
& \left(\operatorname{ker} D_{\alpha}^{-}\right)_{\alpha \in \hat{\Gamma}} \rightarrow \hat{\Gamma} .
\end{aligned}
$$

These are indeed well-defined subfields of $\left(H_{\alpha}\right)_{\alpha \in \hat{\Gamma}}$ because $\operatorname{ker} D_{\alpha}^{ \pm}=\operatorname{ker} F_{\alpha}^{ \pm}$by the elliptic regularity theorem.

Suppose that $\left(\operatorname{ker} D_{\alpha}\right)_{\alpha \in \hat{\Gamma}}$ and $\left(\operatorname{coker} D_{\alpha}\right)_{\alpha \in \hat{\Gamma}}$ are vector bundles over $\hat{\Gamma}$ in the relative topology. As in the proof of the Atiyah-Jänich Theorem (cf. [72]), the operator $D$ can always be replaced by another operator in such a way that the class $\left[L^{2}(M, E), F\right]$ does not change, and that these 'fields of vector spaces' are indeed vector bundles (see also [35]). Then:

Proposition 5.3. The image of the class $\left[L^{2}(M, E), F\right] \in K_{0}^{\Gamma}(M)$ under the assembly map $\mu_{M}^{\Gamma}$ is

$$
\mu_{M}^{\Gamma}\left[L^{2}(M, E), F\right]=\left[\left(\operatorname{ker} D_{\alpha}^{+}\right)_{\alpha \in \hat{\Gamma}}\right]-\left[\left(\operatorname{ker} D_{\alpha}^{-}\right)_{\alpha \in \hat{\Gamma}}\right] \in K^{0}(\hat{\Gamma}) .
$$

Proposition 5.3 wil be proved in the next two subsections.

\subsection{The Hilbert $C^{*}$-module part of the assembly map}

In this subsection we determine the Hilbert $C^{*}(\Gamma) \cong C_{0}(\hat{\Gamma})$-module $\mathcal{E}$ in (48) (Proposition 5.77).

\section{A unitary isomorphism}

Let $d \alpha$ be the measure on $\hat{\Gamma}$ corresponding to the counting measure on $\Gamma$ via the Fourier transform. Consider the Hilbert space

$$
H:=\int_{\hat{\Gamma}}^{\oplus} H_{\alpha} d \alpha
$$

That is, $H$ consists of the measurable maps

$$
s: \hat{\Gamma} \rightarrow\left(H_{\alpha}\right)_{\alpha \in \hat{\Gamma}}, \quad \alpha \mapsto s_{\alpha},
$$

such that $s_{\alpha} \in H_{\alpha}$ for all $\alpha$, and

$$
\|s\|_{H}^{2}=\langle s, s\rangle_{H}:=\int_{\hat{\Gamma}}\left\|s_{\alpha}\right\|_{\alpha}^{2} d \alpha<\infty .
$$

Define the linear map $V: H \rightarrow L^{2}(M, E)$ by

$$
(V s)(m):=\int_{\hat{\Gamma}} s_{\alpha}(m) d \alpha .
$$

Lemma 5.4. The map $V$ is a unitary isomorphism, with inverse

$$
\left(V^{-1} \sigma\right)_{\alpha}(m)=\sum_{\gamma \in \Gamma} \gamma \cdot \sigma\left(\gamma^{-1} m\right) U_{\alpha}(\gamma),
$$

for all $\sigma \in C_{c}(M, E) \subset L^{2}(M, E)$. 
Remark 5.5. It follows from unitarity of $V$ that $V s$ is indeed an $L^{2}$-section of $E$ for all $s \in H$. Conversely, a direct computation shows that for all $\sigma \in L^{2}(M, E), \alpha \in \hat{\Gamma}$ and $\gamma \in \Gamma$, one has

$$
\gamma \cdot\left(V^{-1} \sigma\right)_{\alpha}=U_{\alpha}(\gamma)^{-1}\left(V^{-1} \sigma\right)_{\alpha},
$$

so that $V^{-1} \sigma$ lies in $H$.

Sketch of proof of Lemma 5.4. The proof is based on the observations that for all $\alpha \in \hat{\Gamma}$,

$$
\sum_{\gamma \in \Gamma} U_{\alpha}(\gamma)=\delta_{1}(\alpha)
$$

where $\delta_{1} \in \mathcal{D}^{\prime}(\hat{\Gamma})$ is the $\delta$-distribution at the trivial representation $1 \in \hat{\Gamma}$, and that for all $\gamma \in \Gamma$,

$$
\int_{\hat{\Gamma}} U_{\alpha}(\gamma) d \alpha=\delta_{\gamma e}
$$

the Kronecker delta of $\gamma$ and the identity element. Using these facts, one can easily verify that $V$ is an isometry and that (52) is indeed the inverse of $V$.

The representation $\pi_{H}$ of $\Gamma$ in $H$ corresponding to the standard representation of $\Gamma$ in $L^{2}(M, E)$ via the isomorphism $V$ is given by

$$
\left(\pi_{H}(\gamma) s\right)_{\alpha}=U_{\alpha}(\gamma)^{-1} s_{\alpha} .
$$

This follows directly from the definitions of the space $H_{\alpha}$ and the map $V$.

\section{Fourier transform}

The Hilbert $C^{*}(\Gamma)$-module $\mathcal{E}$ is the closure of the space $C_{c}(M, E)$ in the norm

$$
\|\sigma\|_{\mathcal{E}}^{2}:=\left\|\gamma \mapsto\langle\sigma, \gamma \cdot \sigma\rangle_{L^{2}(M, E)}\right\|_{C^{*}(\Gamma)} .
$$

The $C^{*}(\Gamma)$-module structure of $\mathcal{E}$ is defined by

$$
f \cdot \sigma=\sum_{\gamma \in \Gamma} f(\gamma) \gamma \cdot \sigma,
$$

for all $f \in C_{c}(\Gamma)$ and $\sigma \in C_{c}(M, E)$. The isomorphism $V$ induces an isomorphism of the Hilbert $C^{*}(\Gamma)$-module $\mathcal{E}$ with the closure $\mathcal{E}_{H}$ of $V^{-1}\left(C_{c}(M, E)\right) \subset H$ in the norm

$$
\|s\|_{\mathcal{E}_{H}}^{2}:=\left\|\gamma \mapsto\langle V s, \gamma \cdot V s\rangle_{L^{2}(M, E)}\right\|_{C^{*}(\Gamma)}=\left\|\gamma \mapsto\left\langle s, \pi_{H}(\gamma) s\right\rangle_{H}\right\|_{C^{*}(\Gamma)},
$$

by unitarity of $V$. The $C^{*}(\Gamma)$-module structure on $\mathcal{E}_{H}$ corresponding to the one on $\mathcal{E}$ via $V$ is given by

$$
f \cdot s=\sum_{\gamma \in \Gamma} f(\gamma) \pi_{H}(\gamma) s
$$

for all $f \in C_{c}(\Gamma)$ and $s \in V^{-1}\left(C_{c}(M, E)\right)$.

Next, we use the isomorphism $C_{0}(\hat{\Gamma}) \cong C^{*}(\Gamma)$ defined by the Fourier transform $\psi \mapsto \hat{\psi}$, where

$$
\hat{\psi}(\gamma)=\int_{\hat{\Gamma}} \psi(\alpha) U_{\alpha}(\gamma) d \alpha
$$

for all $\psi \in C_{c}(\hat{\Gamma})$. Because of (53) and (54), the inverse Fourier transform is given by $f \mapsto \hat{f}$, where for $f \in C_{c}(\Gamma)$, one has

$$
\hat{f}(\alpha)=\sum_{\gamma \in \Gamma} f(\gamma) U_{\alpha}(\gamma)^{-1}
$$

So by Fourier transform, the Hilbert $C^{*}(\Gamma)$-module $\mathcal{E}_{H}$ corresponds to the Hilbert $C_{0}(\hat{\Gamma})$-module $\hat{\mathcal{E}}_{H}$, which is the closure of the space $V^{-1}\left(C_{c}(M, E)\right)$ in the norm

$$
\begin{aligned}
\|s\|_{\hat{\mathcal{E}}_{H}}^{2} & =\left\|\alpha \mapsto \sum_{\gamma \in \Gamma}\left\langle s, \pi_{H}(\gamma) s\right\rangle_{H} U_{\alpha}(\gamma)^{-1}\right\|_{C_{0}(\hat{\Gamma})} \\
& =\sup _{\alpha \in \hat{\Gamma}}\left|\sum_{\gamma \in \Gamma}\left\langle s, \pi_{H}(\gamma) s\right\rangle_{H} U_{\alpha}(\gamma)^{-1}\right| .
\end{aligned}
$$




\section{Continuous sections}

Using the following lemma, we will describe the Hilbert $C_{0}(\hat{\Gamma})$-module $\hat{\mathcal{E}}_{H}$ as the space of continuous sections of a continuous field of Hilbert spaces.

Lemma 5.6. For all $s, t \in V^{-1}\left(C_{c}(M, E)\right)$,

$$
\sum_{\gamma \in \Gamma}\left\langle s, \pi_{H}(\gamma) t\right\rangle_{H} U_{\alpha}(\gamma)^{-1}=\left\langle s_{\alpha}, t_{\alpha}\right\rangle_{\alpha}
$$

Proof. Let $\varphi$ be a measurable section of the principal fibre bundle $M \rightarrow M / \Gamma$. Then

$$
\begin{aligned}
\sum_{\gamma \in \Gamma}\left\langle s, \pi_{H}(\gamma) t\right\rangle_{H} U_{\alpha}(\gamma)^{-1} & =\sum_{\gamma \in \Gamma}\left(\int_{\hat{\Gamma}} \int_{M / \Gamma}\left(s_{\beta}(\varphi(\mathcal{O})), U_{\beta}(\gamma)^{-1} t_{\beta}(\varphi(\mathcal{O}))\right)_{E} d \mathcal{O} d \beta\right) U_{\alpha}(\gamma)^{-1} \\
& =\int_{M / \Gamma}\left(s_{\alpha}(\varphi(\mathcal{O})), t_{\alpha}(\varphi(\mathcal{O}))\right)_{E} d \mathcal{O} \\
& =\left\langle s_{\alpha}, t_{\alpha}\right\rangle_{\alpha} .
\end{aligned}
$$

by (53).

We conclude from (56) and Lemma [5.6] that $\hat{\mathcal{E}}_{H}$ is the closure of $V^{-1}\left(C_{c}(M, E)\right)$ in the norm

$$
\|s\|_{\hat{\mathcal{E}}_{H}}^{2}=\sup _{\alpha \in \hat{\Gamma}}\left\|s_{\alpha}\right\|_{\alpha}^{2}
$$

Therefore, it makes sense to define the space $C\left(\hat{\Gamma},\left(H_{\alpha}\right)_{\alpha \in \hat{\Gamma}}\right)$ of continuous sections of the field of Hilbert spaces $\left(H_{\alpha}\right)_{\alpha \in \hat{\Gamma}}$ as the $C_{0}(\hat{\Gamma})$-module $\hat{\mathcal{E}}_{H}$ (cf. [15, 67]). Then our construction implies

Proposition 5.7. The Hilbert $C^{*}(\Gamma)$-module $\mathcal{E}$ is isomorphic to the Hilbert $C_{0}(\hat{\Gamma})$-module $C\left(\hat{\Gamma},\left(H_{\alpha}\right)_{\alpha \in \hat{\Gamma}}\right)$.

Let us verify explicitly that the representations of $C_{0}(\hat{\Gamma})$ in $\hat{\mathcal{E}}_{H}$ and in $C\left(\hat{\Gamma},\left(H_{\alpha}\right)_{\alpha \in \hat{\Gamma}}\right)$ are indeed intertwined by the isomorphism induced by $V$ and the Fourier transform: for all $f \in C_{c}(\Gamma)$ and all $s \in V^{-1}\left(C_{c}(M, E)\right)$, we have

$$
\begin{aligned}
(f \cdot s)_{\alpha} & =\sum_{\gamma \in \Gamma} f(\gamma)\left(\pi_{H}(\gamma) s\right)_{\alpha} \quad \text { by (55) } \\
& =\sum_{\gamma \in \Gamma} f(\gamma) U_{\alpha}(\gamma)^{-1} s_{\alpha} \\
& =\hat{f}(\alpha) s_{\alpha} .
\end{aligned}
$$

\subsection{The operator part of the assembly map}

Proposition 5.8. The operator $F_{\hat{\mathcal{E}}_{H}}$ on the Hilbert $C_{0}(\hat{\Gamma})$ module $\hat{\mathcal{E}}_{H}=C\left(\hat{\Gamma},\left(H_{\alpha}\right)_{\alpha \in \hat{\Gamma}}\right)$, induced by $F \in \mathcal{B}\left(L^{2}(M, E)\right)$, equals $F_{\hat{\mathcal{E}}_{H}}=\left(F_{\alpha}\right)_{\alpha \in \hat{\Gamma}}$, where the 'field of operators' $\left(F_{\alpha}\right)_{\alpha \in \hat{\Gamma}}$ is given by

$$
\left(\left(F_{\alpha}\right)_{\alpha \in \hat{\Gamma}} s\right)_{\beta}=F_{\beta} s_{\beta},
$$

for all $\beta \in \hat{\Gamma}$ and all $s \in V^{-1}\left(C_{c}(M, E)\right.$ ) (and extended continuously to general $s \in \hat{\mathcal{E}}_{H}$ ). Here $F_{\alpha}$ is the operator (51).

Proof. For $s \in V^{-1}\left(C_{c}(M, E)\right)$, we have $V F_{\hat{\mathcal{E}}_{H}} s=F V s$. So it is sufficient to prove that for such $s$, one has $F V s(m)=\int_{\hat{\Gamma}} F_{\alpha} s_{\alpha}(m) d \alpha$, for all $m \in M$.

Let $H^{D} \subset H$ be the space of $s \in H$ such that $V s \in C_{c}^{\infty}(M, E)$, and $s_{\alpha} \in H_{\alpha}^{D}$ for all $\alpha \in \hat{\Gamma}$ (see (501). By Proposition 3.10 we have $D V s(m)=\int_{\hat{\Gamma}} D s_{\alpha}(m) d \alpha$ for all $s \in H^{D}$ and $m \in M$. Because of Lemma 3.4 this proves the proposition, because $H^{D}$ is dense in $H$. 
Note that $\left(F_{\alpha}\right)_{\alpha \in \hat{\Gamma}}$ is initially defined on the subspace $V^{-1}\left(C_{c}(M, E)\right)$ of $C\left(\hat{\Gamma},\left(H_{\alpha}\right)_{\alpha \in \hat{\Gamma}}\right)$. But since the unitary operator $V$ intertwines $\left(F_{\alpha}\right)_{\alpha \in \hat{\Gamma}}$ and the bounded operator $F$ on $L^{2}(M, E)$, the field of operators $\left(F_{\alpha}\right)_{\alpha \in \hat{\Gamma}}$ is bounded in the norm $\|\cdot\|_{\hat{\mathcal{E}}_{H}}$, so that it extends continuously to a bounded operator on $C\left(\hat{\Gamma},\left(H_{\alpha}\right)_{\alpha \in \hat{\Gamma}}\right)$.

Proof of Proposition 5.3. We have seen (cf. Propositions 5.7 and 5.8) that

$$
\mu_{M}^{\Gamma}\left[L^{2}(M, E), F\right]=\left[C\left(\hat{\Gamma},\left(H_{\alpha}\right)_{\alpha \in \hat{\Gamma}}\right),\left(F_{\alpha}\right)_{\alpha \in \hat{\Gamma}}\right] \in K K_{0}\left(\mathbb{C}, C_{0}(\hat{\Gamma})\right) .
$$

The image of this class in $K_{0}\left(C_{0}(\hat{\Gamma})\right)$ is the formal difference of projective $C_{0}(\hat{\Gamma})$-modules

$$
\left[\operatorname{ker}\left(\left(F_{\alpha}^{+}\right)_{\alpha \in \hat{\Gamma}}\right)\right]-\left[\operatorname{ker}\left(\left(F_{\alpha}^{-}\right)_{\alpha \in \hat{\Gamma}}\right)\right] .
$$

By compactness of $M / \Gamma$ and the elliptic regularity theorem, the kernels of $F_{\alpha}^{+}$and $F_{\alpha}^{-}$are equal to the kernels of $D_{\alpha}^{+}$and $D_{\alpha}^{-}$, respectively. If we suppose that the kernels of $D_{\alpha}^{+}$and $D_{\alpha}^{-}$define vector bundles over $\hat{\Gamma}$, then by Lemma 5.9 below, the class (57) equals

$$
\left[C\left(\hat{\Gamma},\left(\operatorname{ker} D_{\alpha}^{+}\right)_{\alpha \in \hat{\Gamma}}\right)\right]-\left[C\left(\hat{\Gamma},\left(\operatorname{ker} D_{\alpha}^{-}\right)_{\alpha \in \hat{\Gamma}}\right)\right] .
$$

Under the isomorphism $K_{0}\left(C_{0}(\hat{\Gamma})\right) \cong K^{0}(\hat{\Gamma})$, the latter class corresponds to

$$
\left[\left(\operatorname{ker} D_{\alpha}^{+}\right)_{\alpha \in \hat{\Gamma}}\right]-\left[\left(\operatorname{ker} D_{\alpha}^{-}\right)_{\alpha \in \hat{\Gamma}}\right] \in K^{0}(\hat{\Gamma}) \text {. }
$$

Lemma 5.9. Let $\mathcal{H}$ be a continuous field of Hilbert spaces over a topological space $X$, and let $\Delta$ be its space of continuous sections. Let $\mathcal{H}^{\prime}$ be a subset of $\mathcal{H}$ such that for all $x \in X, \mathcal{H}_{x}^{\prime}:=\mathcal{H}_{x} \cap \mathcal{H}^{\prime}$ is a linear subspace of $\mathcal{H}_{x}$. Set

$$
\Delta^{\prime}:=\left\{s \in \Delta ; s(x) \in \mathcal{H}_{x}^{\prime} \text { for all } x \in X\right\} .
$$

Let $s: X \rightarrow \mathcal{H}^{\prime}$ be a section. Then $s$ is continuous in the subspace topology of $\mathcal{H}^{\prime}$ in $\mathcal{H}$ if and only if $s \in \Delta^{\prime}$.

Proof. Let $s: X \rightarrow \mathcal{H}$ be a section. Then $s$ is a continuous section of $\mathcal{H}^{\prime}$ in the subspace topology if and only if $s$ is a continuous section of $\mathcal{H}$ and $s(x) \in \mathcal{H}_{x}^{\prime}$ for all $x$. The topology on $\mathcal{H}$ is defined in such a way that $s$ is continuous if and only if $s \in \Delta$ [15, 67.

\subsection{Reduction}

We will now describe the reduction map $R_{Q}^{(\Gamma)}: K_{0}\left(C^{*}(\Gamma)\right) \rightarrow \mathbb{Z}$, and prove Proposition 5.1

Lemma 5.10. Let $\Gamma$ be an abelian discrete group, and let $i:\{1\} \hookrightarrow \hat{\Gamma}$ be the inclusion of the trivial representation. The following diagram commutes:

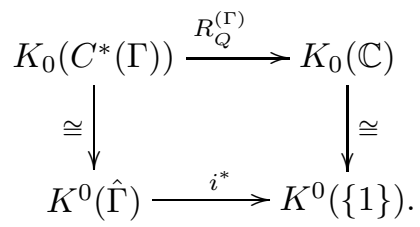

That is,

$$
R_{Q}^{(\Gamma)}([E])=\operatorname{dim} E_{1}=\operatorname{rank}(E) \in \mathbb{Z},
$$

for all vector bundles $E \rightarrow \hat{\Gamma}$. 
The proof is a straightforward verification.

End of proof of Proposition 5.1. From Lemma 5.10 and Proposition 5.3] we conclude that

$$
R_{Q}^{(\Gamma)} \circ \mu_{M}^{\Gamma}\left[L^{2}(M, E), F\right]=\left[\operatorname{ker} D_{1}^{+}\right]-\left[\operatorname{ker} D_{1}^{-}\right]=\operatorname{index} D_{1} \in \mathbb{Z} .
$$

The Hilbert space $H_{1}$ is isomorphic to $L^{2}(M / \Gamma, E / \Gamma)$, and this isomorphism intertwines $D_{1}$ and $D^{\Gamma}$. Hence Proposition 5.1 follows.

\section{Example: action of $\mathbb{Z}^{2 n}$ on $\mathbb{R}^{2 n}$}

Let $M$ be the manifold $M=T^{*} \mathbb{R}^{n} \cong \mathbb{R}^{2 n} \cong \mathbb{C}^{n}$. An element of $M$ is denoted by $(q, p):=$ $\left(q_{1}, p_{1}, \ldots, q_{n}, p_{n}\right)$, where $q_{j}, p_{j} \in \mathbb{R}$, or by $q+i p=z:=\left(z_{1}, \ldots, z_{n}\right)$, where $z_{j}=q_{j}+i p_{j} \in \mathbb{C}$. We equip $M$ with the standard symplectic form $\omega:=\sum_{j=1}^{n} d p_{j} \wedge d q_{j}$.

Let $\Gamma$ be the group $\Gamma=\mathbb{Z}^{2 n} \cong \mathbb{Z}+i \mathbb{Z}$. The action of $\Gamma$ on $M$ by addition is denoted by $\alpha$. Our aim is to find a prequantisation for this action and the corresponding Dirac operator for general $n$, and the quantisation of this action for $n=1$. This is less trivial than it may seem because of the coupling of the standard Dirac operator to the prequantum line bundle, which precludes the use of the standard formulae. We will then see, just as in Section 5 that the reduction of this quantisation is the quantisation of the reduced space $\mathbb{T}^{2}$.

\subsection{Prequantisation}

Let $L:=M \times \mathbb{C} \rightarrow M$ be the trivial line bundle. Inspired by the construction of line bundles on tori with a given Chern class (see e.g. 24], pp. 307-317), we lift the action of $\Gamma$ on $M$ to an action of $\Gamma$ on $L$ (still called $\alpha$ ), by setting

$$
\begin{aligned}
e_{j} \cdot(z, w) & =\left(z+e_{j}, w\right) ; \\
i e_{j} \cdot(z, w) & =\left(z+i e_{j}, e^{-2 \pi i z_{j}} w\right) .
\end{aligned}
$$

Here $z \in M, w \in \mathbb{C}$, and

$$
e_{j}:=(0, \ldots, 0,1,0, \ldots, 0) \in \mathbb{Z}^{n},
$$

the 1 being in the $j^{\text {th }}$ place. The corresponding representation of $\Gamma$ in the space of smooth sections of $L$ is denoted by $\rho$ :

$$
\left(\rho_{k+i l} s\right)(z)=\alpha_{k+i l} s(z-k-i l),
$$

for $k, l \in \mathbb{Z}^{n}$ and $z \in M$. Define the metric $H$ on $L$ by

$$
H\left((z, w),\left(z, w^{\prime}\right)\right)=h(z) w \bar{w}^{\prime},
$$

where $z \in M, w, w^{\prime} \in \mathbb{C}$, and $h \in C^{\infty}(M)$ is defined by

$$
h(q+i p):=e^{2 \pi \sum_{j}\left(p_{j}-p_{j}^{2}\right)} .
$$

Let $\nabla$ be the connection on $L$ defined by

$$
\nabla:=d+2 \pi i \sum_{j=1}^{n} p_{j} d z_{j}+\pi d p_{j}
$$

Proposition 6.1. The triple $(L, H, \nabla)$ defines an equivariant prequantisation for $(M, \omega)$.

The proof of this proposition is a set of tedious computations. Because of the term $2 \pi i \sum_{j=1}^{n} p_{j} d q_{j}$ in the expression for the connection $\nabla$, it has the right curvature form. The terms $-2 \pi \sum_{j=1}^{n} p_{j} d p_{j}$ and $\pi d p_{j}$ do not change the curvature, and have been added to make $\nabla$ equivariant. At the same time, the latter two terms ensure that there is a $\Gamma$-invariant metric (namely $H$ ) with respect to which $\nabla$ is Hermitian. 
As we mentioned in Subsection 2.1] there is a procedure in [27 to lift the action of $\mathbb{Z}^{2 n}$ on $\mathbb{R}^{2 n}$ to a projective action on $L$ that leaves the connection (for example) $\nabla^{\prime}:=d+2 \pi i \sum_{j} p_{j} d q_{j}$ invariant. This projective action turns out to be an actual action in this case, and preserves the standard metric on $L$. We thus obtain prequantisation of this action that looks much simpler than the one given in this section. However, we found our formulas to be more suitable to compute the kernel of the associated Dirac operator.

\subsection{The Dirac operator}

In this subsection, we compute the Dolbeault-Dirac operator $\not D$ on $M$, coupled to $L$. To compute the quantisation of the action we are considering, we need to compute the kernels of

$$
\begin{aligned}
& \not D^{+}:=\left.\not D\right|_{\Omega^{0, \mathrm{even}}(M)} ; \\
& \not D^{-}:=\left.\not D\right|_{\Omega^{0, \text { odd }}(M)} .
\end{aligned}
$$

This is not easy to do in general. But for $n=1$, these kernels are computed in Subsection 6.3

In our expression for the Dirac operator, we will use multi-indices

$$
l=\left(l_{1}, \ldots, l_{q}\right) \subset\{1, \ldots, n\},
$$

where $q \in\{0, \ldots, n\}$ and $l_{1}<\cdots<l_{q}$. We will write $d \bar{z}^{l}:=d \bar{z}_{l_{1}} \wedge \ldots \wedge d \bar{z}_{l_{q}}$. If $l=\emptyset$, we set $d \bar{z}^{l}:=1_{M}$, the constant function 1 on $M$. Note that $\left\{d \bar{z}^{l}\right\}_{l \subset\{1, \ldots, n\}}$ is a $C^{\infty}(M)$-basis of $\Omega^{0, *}(M ; L)$.

Given $l \subset\{1, \ldots, n\}$ and $j \in\{1, \ldots, n\}$, we define

$$
\varepsilon_{j l}:=(-1)^{\#\left\{r \in\{1, \ldots, q\} ; l_{r}<j\right\}},
$$

plus one if an even number of $l_{r}$ is smaller than $j$, and minus one if the number of such $l_{r}$ is odd. From the definition of the Dolbeault-Dirac operator one then deduces:

Proposition 6.2. For all $l \subset\{1, \ldots, n\}$ and all $f \in C^{\infty}(M)$, we have

$$
\begin{gathered}
\not D\left(f d \bar{z}^{l}\right)=\sum_{j \in l} \varepsilon_{j l}\left(-2 \frac{\partial f}{\partial z_{j}}+\left(i \pi-4 \pi i p_{j}\right) f\right) d \bar{z}^{l \backslash\{j\}} \\
+\sum_{\substack{1 \leq j \leq n, j \notin l}} \varepsilon_{j l}\left(\frac{\partial f}{\partial \bar{z}_{j}}+\frac{i \pi}{2} f\right) d \bar{z}^{l \cup\{j\}} .
\end{gathered}
$$

\subsection{The case $n=1$}

We now consider the case where $n=1$. That is, $M=\mathbb{C}$ and $\Gamma=\mathbb{Z}+i \mathbb{Z}$. We can then explicitly compute the quantisation of the action. This will allow us to illustrate our noncompact GuilleminSternberg conjecture by computing the four corners in diagram (11).

If $n=1$, Proposition 6.2 reduces to

Corollary 6.3. The Dirac operator on $\mathbb{C}$, coupled to $L$, is given by

$$
\not D\left(f_{1}+f_{2} d \bar{z}\right)=\left(\frac{\partial f_{1}}{\partial \bar{z}}+\frac{i \pi}{2} f_{1}\right) d \bar{z}-2 \frac{\partial f_{2}}{\partial z}+(i \pi-4 \pi i p) f_{2} .
$$

That is to say, with respect to the $C^{\infty}(M)$-basis $\left\{1_{M}, d \bar{z}\right\}$ of $\Omega^{0, *}(M ; L)$, the Dirac operator $\not D$ has

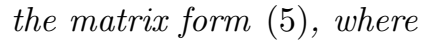

$$
\begin{aligned}
& \not D^{+}=\frac{\partial}{\partial \bar{z}}+\frac{i \pi}{2} \\
& \not D^{-}=-2 \frac{\partial}{\partial z}+i \pi-4 \pi i p .
\end{aligned}
$$


In this case, the kernels of $\not D^{+}$and $\not D^{-}$can be determined explicitly:

Proposition 6.4. The kernel of $\not D^{+}$consists of the sections $s$ of $L$ given by

$$
s(z)=e^{-i \pi \bar{z} / 2} \varphi(z)
$$

where $\varphi$ is a holomorphic function.

The kernel of $\not D^{-}$is isomorphic to the space of smooth sections $t$ of $L$ given by

$$
t(z)=e^{i \pi z / 2+\pi|z|^{2}-\pi z^{2} / 2} \overline{\psi(z)},
$$

where $\psi$ is a holomorphic function.

The unitary dual of the group $\mathbb{Z}+i \mathbb{Z}=\mathbb{Z}^{2}$ is the torus $\mathbb{T}^{2}$. Therefore, by Proposition 5.3 the quantisation of the action of $\mathbb{Z}+i \mathbb{Z}$ on $\mathbb{C}$ is the class in $K K\left(\mathbb{C}, C^{*}\left(\mathbb{Z}^{2}\right)\right)$ that corresponds to the class

$$
\left[\left(\operatorname{ker} \not D_{(\alpha, \beta)}^{+}\right)_{(\alpha, \beta) \in \mathbb{T}^{2}}\right]-\left[\left(\operatorname{ker} \not D_{(\alpha, \beta)}^{-}\right)_{(\alpha, \beta) \in \mathbb{T}^{2}}\right]
$$

in $K^{0}\left(\mathbb{T}^{2}\right)$. It will turn out that the kernels of $\not D_{(\alpha, \beta)}^{+}$and $\not D_{(\alpha, \beta)}^{-}$indeed define vector bundles over $\mathbb{T}^{2}$. Let us compute these kernels.

Proposition 6.5. Let $\lambda, \mu \in \mathbb{R}$. Define the section $s_{\lambda \mu} \in C^{\infty}(M, L)$ by

$$
s_{\lambda \mu}(z)=e^{i \lambda z} e^{-\pi p} \sum_{k \in \mathbb{Z}} e^{-\pi k^{2}} e^{-k(\lambda+i \mu+2 \pi)} e^{2 \pi i k z} .
$$

Set $\alpha:=e^{i \lambda}$ and $\beta:=e^{i \mu}$. Then $\operatorname{ker} \not D_{(\alpha, \beta)}^{+}=\mathbb{C} s_{\lambda \mu}$.

Remark 6.6. For all $\lambda, \mu \in \mathbb{R}$, we have

$$
\begin{aligned}
& s_{\lambda+2 \pi, \mu}=e^{\lambda+i \mu+3 \pi} s_{\lambda \mu} ; \\
& s_{\lambda, \mu+2 \pi}=s_{\lambda \mu} .
\end{aligned}
$$

Hence the vector space $\mathbb{C} s_{\lambda \mu} \subset C^{\infty}(M, L)$ is invariant under $\lambda \mapsto \lambda+2 \pi$ and $\mu \mapsto \mu+2 \pi$. This is in agreement with the fact that $\mathbb{C} s_{\lambda \mu}$ is the kernel of $\not D_{\left(e^{i \lambda}, e^{i \mu}\right)}^{+}$.

Sketch of proof of Proposition 6.5. Let $\lambda, \mu \in \mathbb{R}$, and $s \in C^{\infty}(M, L)=C^{\infty}(\mathbb{C}, \mathbb{C})$. Suppose $s$ is in the kernel of $\not D_{(\alpha, \beta)}^{+}$. Let $\varphi$ be the holomorphic function from Proposition 6.4 and write

$$
\tilde{\varphi}(z):=e^{-i \lambda z} e^{-i \pi z / 2} \varphi(z)=\sum_{k \in \mathbb{Z}} a_{k} e^{2 \pi i k z}
$$

(note that for all $z \in \mathbb{C}$ one has $\tilde{\varphi}(z+1)=\tilde{\varphi}(z))$. Then $a_{k}=e^{-\pi k^{2}} e^{-k(\lambda+i \mu+2 \pi)} a_{0}$, which gives the desired result.

Proposition 6.7. The kernel of $\not D_{(\alpha, \beta)}^{-}$is trivial for all $(\alpha, \beta) \in \mathbb{T}^{2}$.

Sketch of proof. Let $\lambda, \mu \in \mathbb{R}$ and let $t d \bar{z} \in \Omega^{0,1}(M ; L)=C^{\infty}(M, L) d \bar{z}$. Suppose that $t d \bar{z} \in$ $\operatorname{ker} \not D_{\left(e^{i \lambda}, e^{i \mu}\right)}^{-}$. Let $\psi$ be the holomorphic function from Proposition 6.4 and write

$$
\tilde{\psi}(z):=e^{\pi\left(\bar{z}^{2}+i \bar{z}\right) / 2-i \lambda \bar{z}} \overline{\psi(z)}=\sum_{k \in \mathbb{Z}} c_{k} e^{2 \pi i k \bar{z}}
$$

(note that for all $z \in \mathbb{C}$ one has $\tilde{\psi}(z+1)=\tilde{\psi}(z)$ ). Then $c_{k}=e^{\pi k^{2}} e^{k(\lambda-i \mu-2 \pi)} c_{0}$, which implies that $c_{0}=0$.

We conclude: 
Proposition 6.8. The quantisation of the action of $\mathbb{Z}^{2}$ on $\mathbb{C}$ is the class in $K^{0}\left(\mathbb{T}^{2}\right)$ defined by the vector bundle 28

$$
\left(\mathbb{C} s_{\lambda \mu}\right)_{\left(e^{i \lambda}, e^{i \mu}\right) \in \mathbb{T}^{2}} \rightarrow \mathbb{T}^{2} .
$$

By Lemma 5.10 we now find that the reduction of the quantisation of the action of $\mathbb{Z}^{2}$ on $\mathbb{R}^{2}$ is the one-dimensional vector space $\mathbb{C} \cdot s_{0,0} \subset C^{\infty}(M, L)$, where

$$
s_{0,0}(z)=e^{-\pi p} \sum_{k \in \mathbb{Z}} e^{-\pi k^{2}} e^{-2 \pi k} e^{2 \pi i k z} .
$$

As we saw in Section 5.1 this is precisely the index of $\not D_{\mathbb{T}^{2}}^{L / \mathbb{Z}^{2}}$. Schematically, we therefore have ${ }^{29}$

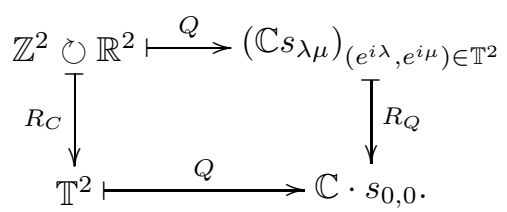

Remark 6.9. The fact that the geometric quantisation of the torus $\mathbb{T}^{2}$ is one-dimensional can alternatively be deduced from the Atiyah-Singer index theorem for Dirac operators. Indeed, let $\not D_{\mathbb{T}^{2}}^{L / \mathbb{Z}^{2}}$ be the Dirac operator on the torus, coupled to the quotient line bundle $L / \mathbb{Z}^{2}$. Then by Atiyah-Singer, in the form stated for example in [25] on page 117, one has

$$
\begin{aligned}
Q\left(\mathbb{T}^{2}\right)=\operatorname{index} \not D_{\mathbb{T}^{2}}^{L / \mathbb{Z}^{2}} & =\int_{\mathbb{T}^{2}} e^{\operatorname{ch}_{1}\left(L / \mathbb{Z}^{2}\right)} \\
& =\int_{\mathbb{T}^{2}} d p \wedge d q \\
& =1
\end{aligned}
$$

the symplectic volume of the torus, i.e. the volume determined by the Liouville measure.

\section{A Naturality of the assembly map}

Our "quantisation commutes with reduction" result is partly a consequence of the naturality of the assembly map. For discrete groups, this naturality is explained in detail by Valette [52]. We need to generalise "one half" of this naturality (the epimorphism case) to non-discrete groups.

\section{A.1 The statement}

Let $G$ be a locally compact unimodular group acting properly on a locally compact Hausdorff space $X$. We consider a closed normal subgroup ${ }^{30} N$ of $G$, and suppose that $G$ and $N$ are equipped with Haar measures $d g$ and $d n$, respectively. We suppose that $X / G$ is compact.

The version of naturality of the assembly map that we will need is the following.

Theorem A.1. The homomorphism $V_{N}$ defined in Subsection 3.1 makes the following diagram commute:

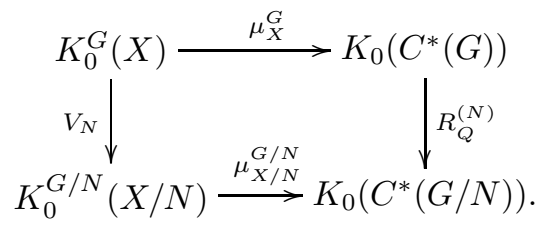

\footnotetext{
${ }^{28}$ By Remark 6.6 this is indeed a well-defined vector bundle.

${ }^{29}$ Note that it is a coincidence that the two-torus appears twice in this diagram: in this example $M / \Gamma=\mathbb{T}^{2}=\hat{\Gamma}$.

${ }^{30}$ Although for our purpose it is enough to consider discrete normal subgroups of $G$, we have to work in the nondiscrete setting anyway, since $G$ is not necessarily discrete. We therefore allow nondiscrete subgroups $N$.
} 
Here $\mu_{X}^{G}$ and $\mu_{X / N}^{G / N}$ are analytic assembly maps as defined in e.g. [5] [0, 52, and the map

$$
R_{Q}^{(N)}=\left(\int_{N}\right)_{*}
$$

is functorially induced by the map $\int_{N}: C^{*}(G) \rightarrow C^{*}(G / N)$ given on $f \in C_{c}(G)$ by 23

$$
\int_{N}(f): N g \mapsto \int_{N} f(n g) d n .
$$

To prove Theorem A.1 one can simply copy the proof for discrete groups in Valette [52, replacing discrete groups by possibly non-discrete ones and sums by integrals. In places where Valette uses the fact that a finite sum of compact operators is again compact, one uses Lemma A.2 This lemma states that in some cases, the integral over a compact set of a continuous family of compact operators is compact.

Another difference between the discrete and the nondiscrete cases is the definition of the map $\Psi$ on page 110 of [52]. In the nondiscrete case this map is defined as follows. If $\xi \in H_{c}$, we will write $\xi^{N}:=\xi+\operatorname{ker}(\cdot, \cdot)_{N}$ for its class in $H_{N}$. Then for all $\xi \in H_{c}$, we have $\xi^{N} \in H_{N, c}$. Define the linear map

$$
\Psi: H_{c} \otimes_{C_{c}(G)} C_{c}(G / N) \rightarrow H_{N}
$$

by

$$
\Psi[\xi \otimes \varphi]=\int_{G / N} \varphi\left(N g^{-1}\right) N g \cdot \xi^{N} d(N g),
$$

where $d(N g)$ is the Haar measure on $G / N$ corresponding to the Haar measures $d n$ and $d g$ on $N$ and $G$, respectively. To prove that the extension $\Psi: \mathcal{E} \otimes_{C^{*}(G)} C^{*}(G / N) \rightarrow \tilde{\mathcal{E}}$ is surjective, one can use a sequence of compactly supported continuous functions on $G / N$ that converges to the distribution $\delta_{N e} \in \mathcal{D}^{\prime}(G / N)$ with respect to the measure $d(N g)$.

\section{A.2 Integrals of families of operators}

Lemma A.2. Let $\mathcal{E}$ be a Hilbert $C^{*}$-module, and let $\mathcal{F}(\mathcal{E})$ and $\mathcal{K}(\mathcal{E})=\overline{\mathcal{F}(\mathcal{E})}$ be the algebras of finite-rank and compact operators on $\mathcal{E}$, respectively. Let $(M, \mu)$ be a compact Borel space with finite measure. Suppose $M$ is metrisable. Let $\alpha, \beta: M \rightarrow \mathcal{B}(\mathcal{E})$ be continous, and let $T \in \mathcal{K}(\mathcal{E})$ be a compact operator. Define the map $\phi: M \rightarrow \mathcal{K}(\mathcal{E})$ by $\phi(m)=\alpha(m) T \beta(m)$. Then the integral $\int_{M} \phi(m) d m$ defines a compact operator on $\mathcal{E}$.

We will prove this lemma in several steps. For continuous maps $\psi: M \rightarrow \mathcal{B}(\mathcal{E})$, we will use the norm

$$
\|\psi\|_{\infty}:=\sup _{m \in M}\|\psi(m)\|_{\mathcal{B}(\mathcal{E})} .
$$

Lemma A.3. Let $(M, \mu)$ be a compact Borel space with finite measure, and let $\mathcal{E}$ be a Hilbert $C^{*}$-module. Let $\phi: M \rightarrow \mathcal{K}(\mathcal{E})$ be a continuous map. Suppose that $\phi$ is 'uniformly compact', in the sense that there exists a sequence $\left(\phi_{j}\right)_{j=1}^{\infty}: M \rightarrow \mathcal{F}(\mathcal{E})$ such that $\left\|\phi_{j}-\phi\right\|_{\infty}$ tends to zero as $j \rightarrow \infty$. Suppose furthermore that for every $j \in \mathbb{N}$, there is a sequence $\left(\phi_{j}^{k}\right)_{k=1}^{\infty}: M \rightarrow \mathcal{F}(\mathcal{E})$ of simple functions (i.e. having finitely many values), such that for all $\varepsilon>0$ there is an $n \in \mathbb{N}$ such that for all $j, k \geq n,\left\|\phi_{j}^{k}-\phi_{j}\right\|<\varepsilon$. Then the integral $\int_{M} \phi(m) d \mu(m)$ defines a compact operator on $\mathcal{E}$.

Proof. For all $j, k \in \mathbb{N}$, the integral $\int_{M} \phi_{j}^{k}(m) d \mu(m)$ is a finite sum of finite-rank operators, and hence a finite-rank operator itself. And because $\left\|\phi_{j}^{j}-\phi\right\|_{\infty} \rightarrow 0$ as $j$ tends to $\infty$, we have

$$
\int_{M} \phi_{j}^{j}(m) d \mu(m) \rightarrow \int_{M} \phi(m) d \mu(m)
$$

in $\mathcal{B}(\mathcal{E})$. Hence $\int_{M} \phi(m) d \mu(m)$ is a compact operator. 
Lemma A.4. In the situation of Lemma A.2 the conditions of Lemma A.9 are satisfied..

Proof. Choose a sequence $\left(T_{j}\right)_{j=1}^{\infty}$ in $\mathcal{F}(\mathcal{E})$ that converges to $T$. For $m \in M$, set

$$
\phi_{j}(m)=\alpha(m) T_{j} \beta(m)
$$

Then

$$
\left\|\phi_{j}-\phi\right\|_{\infty} \leq\|\alpha\|_{\infty}\left\|T_{j}-T\right\|_{\mathcal{B}(\mathcal{E})}\|\beta\|_{\infty} \rightarrow 0
$$

as $j \rightarrow \infty$. Note that $\alpha$ and $\beta$ are continuous functions on a compact space, so their sup-norms are bounded.

Choose sequences of simple functions $\alpha^{k}, \beta^{k}: M \rightarrow \mathcal{B}(\mathcal{E})$ such that $\left\|\alpha^{k}-\alpha\right\|_{\infty} \rightarrow 0$ and $\left\|\beta^{k}-\beta\right\|_{\infty} \rightarrow 0$ as $j$ goes to $\infty$ (see Lemma A.5 below). For all $j, k \in \mathbb{N}$, set

$$
\phi_{j}^{k}(m):=\alpha^{k}(m) T_{j} \beta^{k}(m)
$$

for $m \in M$. Note that

$$
\begin{aligned}
&\left\|\phi_{j}^{k}-\phi_{j}\right\|_{\infty}= \sup _{m \in M}\left\|\alpha^{k}(m) T_{j} \beta^{k}(m)-\alpha(m) T_{j} \beta(m)\right\| \\
&= \sup _{m \in M}\left(\left\|\alpha^{k}(m) T_{j} \beta^{k}(m)-\alpha^{k}(m) T_{j} \beta(m)\right\|\right. \\
&\left.\quad+\left\|\alpha^{k}(m) T_{j} \beta(m)-\alpha(m) T_{j} \beta(m)\right\|\right) \\
& \leq\left\|\alpha^{k}\right\|_{\infty}\left\|T_{j}\right\|\left\|\beta^{k}-\beta\right\|_{\infty}+\left\|\alpha^{k}-\alpha\right\|_{\infty}\left\|T_{j}\right\|\|\beta\|_{\infty} .
\end{aligned}
$$

The sequences $k \mapsto\left\|\alpha^{k}\right\|_{\infty}$ and $j \mapsto\left\|T_{j}\right\|$ are bounded, since $\alpha^{k} \rightarrow \alpha$ and $T_{j} \rightarrow T$. Hence, because the sequences $\left\|\alpha^{k}-\alpha\right\|_{\infty}$ and $\left\|\beta^{k}-\beta\right\|_{\infty}$ tend to zero, we see that $\left\|\phi_{j}^{k}-\phi_{j}\right\|$ can be made smaller than any $\varepsilon>0$ for $k$ large enough, uniformly in $j$.

Lemma A.5. Let $(M, \mu)$ be a metrisable compact Borel space with metric $d_{M}$, let $Y$ be a normed vector space, and let $\alpha: M \rightarrow Y$ be a continuous map.

Then there exists a sequence of simple maps $\alpha^{k}: M \rightarrow Y$ such that $\left(\alpha^{k}\right)^{-1}(V)$ is measurable for all $V \subset Y$, and such that the sequence $\left(\left\|\alpha-\alpha^{k}\right\|_{\infty}\right)_{k=1}^{\infty}$ goes to zero as $k$ goes to infinity.

Proof. For every $k \in \mathbb{N}$, choose a finite covering $\tilde{U}_{k}=\left\{\tilde{V}_{k}^{1}, \ldots, \tilde{V}_{k}^{n_{k}}\right\}$ of $M$ by balls of radius $\frac{1}{k}$. From each $\tilde{U}_{k}$, we construct a partition $U_{k}=\left\{V_{k}^{1}, \ldots, V_{k}^{n_{k}}\right\}$ of $M$, by setting $V_{k}^{1}:=\tilde{V}_{k}^{1}$, and $V_{k}^{j}:=\tilde{V}_{k}^{j} \backslash \bigcup_{i=1}^{j-1} \tilde{V}_{k}^{i}$. for $j=2, \ldots, n_{k}$. Note that the sets $V_{k}^{j}$ are Borel-measurable. For all $k \in \mathbb{N}$ and $j \in\left\{1, \ldots, n_{k}\right\}$, choose an element $m_{k}^{j} \in V_{k}^{j}$. Define the simple map $\alpha^{k}: M \rightarrow Y$ by

$$
\alpha^{k}(m):=\alpha\left(m_{k}^{j}\right) \quad \text { if } m \in V_{k}^{j} .
$$

Note that, because $\alpha$ is continuous (and uniformly continuous because $M$ is compact), for every $\varepsilon>0$ there is a $k_{\varepsilon} \in \mathbb{N}$ such that for all $m, n \in M$,

$$
d_{M}(m, n)<\frac{1}{k_{\varepsilon}} \quad \Rightarrow \quad\|\alpha(m)-\alpha(n)\|_{Y}<\varepsilon .
$$

Hence for all $\varepsilon>0$, all $k>k_{\varepsilon}$, and all $m \in M$ (say $m \in V_{k}^{j}$ ),

$$
\left\|\alpha(m)-\alpha^{k}(m)\right\|_{Y}=\left\|\alpha(m)-\alpha\left(m_{k}^{j}\right)\right\|_{Y}<\varepsilon .
$$

So $\left\|\alpha-\alpha^{k}\right\|_{\infty}$ indeed goes to zero. 


\section{References}

[1] Abraham, R. and J.E. Marsden. Foundations of Mechanics, 2nd ed. Addison Wesley, Redwood City, 1985.

[2] Atiyah, M. Elliptic operators, discrete groups and von Neumann algebras. Soc. Math. France Astérisque 32-33: 43-72, 1976.

[3] Atiyah, M. and W. Schmid. A geometric construction of the discrete series for semisimple Lie groups. Invent. Math. 42: 1-62, 1977.

[4] Baum, P. and A. Connes. Geometric $K$-theory for Lie groups and foliations. Enseign. Math. (2) 46: 3-42, 2000. Preprint from 1982.

[5] Baum, P., A. Connes, and N. Higson. Classifying space for proper actions and $K$-theory of group $C^{*}$-algebras. Contemporary Mathematics 167: 241-291, 1994.

[6] Beals, R. Characterization of pseudodifferential operators and applications. Duke Math. J. 44: 45-57, 1977.

[7] Blackadar, B. K-theory For Operator Algebras. Cambridge University Press, Cambridge, 1998.

[8] Bourbaki, N. Éléments de mathématique, livre VI: Intégration. Hermann, Paris, 1963.

[9] Chabert, J. and S. Echterhoff. Twisted equivariant $K K$-theory and the Baum-Connes conjecture for group extensions. K-Theory 23: 157-200, 2001.

[10] Chabert, J., S. Echterhoff, and R. Nest. The Connes-Kasparov conjecture for almost connected groups. Publ. Math. Inst. Hautes Études Sci. 97: 239-278, 2003.

[11] Connes, A. Noncommutative Geometry. Academic Press, London, 1994.

[12] Connes, A. and H. Moscovici. The $L^{2}$-index theorem for homogeneous spaces of Lie groups. Ann. Math. (2) 115: 291-330, 1982.

[13] Dirac, P.A.M. Lectures on Quantum Mechanics. Belfer School of Science, Yeshiva University, New York, 1964.

[14] Dixmier, J. C $C^{*}$-algebras. North-Holland, Amsterdam, 1977.

[15] Dixmier, J. and A. Douady. Champs continus d'espaces hilbertiens et de $C^{*}$-algèbres. Bulletin de la SMF 91: 227-284, 1963.

[16] Duistermaat, J.J. The Heat Kernel Lefschetz Fixed Point Theorem for the Spin ${ }^{c}$ Dirac Operator. Birkhäuser, Basel, 1996.

[17] Dunford, N. and Schwartz, J. Linear Operators I. Wiley Interscience, New York, 1958.

[18] Duval, C., J. Elhadad, M.J. Gotay, J. Śniatycki, and G.M. Tuynman. Quantization and bosonic BRST theory. Ann. Phys. (N.Y.) 206: 1-26, 1991.

[19] Duval, C., J. Elhadad, and G.M. Tuynman. The BRS method and geometric quantization: some examples. Comm. Math. Phys. 126: 535-557, 1990.

[20] Friedrich, T. Dirac Operators in Riemannian Geometry. American Mathematical Society, Providence, 2000.

[21] Gotay, M.J. Constraints, reduction, and quantization. J. Math. Phys. 27: 2051-2066, 1986.

[22] Gracia-Bondía, J.M., J.C. Várilly, and H. Figueroa. Elements of Noncommutative Geometry. Birkhäuser, Boston, 2001. 
[23] Green, P. $C^{*}$-algebras of transformation groups with smooth orbit space. Pacific J. Math. 72: 71-97, 1977.

[24] Griffiths, P. and J. Harris. Principles of Algebraic Geometry. Wiley, New York, 1978.

[25] Guillemin, V., V. Ginzburg, and Y. Karshon. Moment Maps, Cobordisms, and Hamiltonian Group Actions. American Mathematical Society, Providence, 2002.

[26] Guillemin, V. and Sternberg, S. Geometric quantization and multiplicities of group representations. Invent. Math. 67: 515-538, 1982.

[27] Hawkins, E. Quantization of multiply connected manifolds. Comm. Math. Phys. 255, no. 3: $513-575,2005$.

[28] Heckman, G.J. and P. Hochs. Proc. MRI Spring School 2004: Lie Groups in Analysis, Geometry and Mechanics, E. van den Ban et al. (eds.), to appear.

[29] Henneaux, M. and C. Teitelboim. Quantization of Gauge Systems. Princeton University Press, Princeton, 1992.

[30] Higson, N. Categories of fractions and excision in KK-theory. J. Pure Appl. Algebra 65: 119$138,1990$.

[31] Higson, N. and J. Roe. Analytic K-Homology. Oxford University Press, Oxford, 2000.

[32] Jeffrey, L.C. and F.C. Kirwan. Localization and the quantization conjecture. Topology 36: 647-693, 1997.

[33] Kasparov, G.G. The operator $K$-functor and extensions of $C^{*}$-algebras. Math. USSR Izvestija 16: 513-572, 1981.

[34] Kasparov, G.G. Equivariant KK-theory and the Novikov conjecture. Invent. Math. 91: 147201, 1988.

[35] Lafforgue, V. K-Théorie bivariante pour les algèbres de Banach et conjecture de Baum-Connes. Invent. Math. 149: 2002, 1-95.

[36] Lafforgue, V. Banach KK-theory and the Baum-Connes conjecture. Proceedings of the International Congress of Mathematicians, Vol. II (Beijing, 2002), pp. 795-812. Higher Ed. Press, Beijing, 2002.

[37] Landsman, N.P. Rieffel induction as generalized quantum Marsden-Weinstein reduction. $J$. Geom. Phys. 15: 285-319, 1995.

[38] Landsman, N P. Mathematical Topics Between Classical and Quantum Mechanics. Springer, New York, 1998.

[39] Landsman, N.P. Quantized reduction as a tensor product. Ref. 43, pp. 137-180, 2001. arXiv:math-ph/0008004.

[40] Landsman, N.P. Quantization as a functor. Contemporary Mathematics 315: 9-24, 2002. arXiv:math-ph/0107023.

[41] Landsman, N.P. Deformation quantization and the Baum-Connes conjecture. Comm. Math. Phys. 237: 87-103, 2003. arXiv:math-ph/0210015.

[42] Landsman, N. P. Functorial quantization and the Guillemin-Sternberg conjecture. Twenty Years of Bialowieza: A Mathematical Anthology, pp. 23-45. Ali, S.T., Emch, G.G., Odzijewicz, A., Schlichenmaier, M., and Woronowicz, S.L. (eds). World Scientific, Singapore, 2005. arXiv: math-ph/0307059. 
[43] Landsman, N.P., M. Pflaum, and M. Schlichenmaier (eds.). Quantization of Singular Symplectic Quotients. Birkhäuser, Basel, 2001.

[44] Lusztig, G. Novikov's higher signature and families of elliptic operators. J. Differential Geometry 7: 229-256, 1972.

[45] Marsden, J.E., G. Misiołek, J.-P. Ortega, M. Perlmutter, and T.S. Ratiu. Hamiltonian Reduction by Stages. To appear.

[46] Marsden, J.E. and T.S. Ratiu. Introduction to Mechanics and Symmetry: A Basic Exposition of Classical Mechanical Systems. Springer-Verlag, New York, 1994.

[47] Marsden, J.E. and A. Weinstein. Reduction of symplectic manifolds with symmetry. Rep. Math. Physics 5: 121-130, 1974.

[48] Marsden, J.E. and A. Weinstein. Comments on the history, theory, and applications of symplectic reduction. Ref. [43], pp. 1-19, 2001.

[49] Meinrenken, E. Symplectic surgery and the Spin ${ }^{c}$-Dirac operator. Adv. Math. 134: 240-277, 1998.

[50] Meinrenken, E. and R. Sjamaar. Singular reduction and quantization. Topology 38: 699-761, 1999.

[51] Metzler, D.S. A K-theoretic note on geometric quantization. Manuscripta Math. 100: 277-289, 1999.

[52] Mislin, G. and A. Valette. Proper Group Actions and the Baum-Connes Conjecture. Birkhäuser, Basel, 2000.

[53] Paradan, P.-E. Localization of the Riemann-Roch character. J. Funct. Anal. 187: 442-509, 2001.

[54] Paradan, P.-E. Spin ${ }^{c}$-quantization and the $K$-multiplicities of the discrete series. Ann. Sci. École Norm. Sup. 36, no. 5: 805-845, 2003.

[55] Parthasarathy, R. Dirac operator and the discrete series. Ann. Math. (2) 96: 1-30, 1972.

[56] Pedersen, G.K. $C^{*}$-Algebras and their Automorphism Groups. Academic Press, London, 1979.

[57] Pierrot, F. Une généralisation en $K$-théorie du théorème d'indice $L^{2}$ d'Atiyah Mém. Soc. Math. France 89, 2002.

[58] Reed, M. and B. Simon. Methods of Modern Mathematical Physics, Vol I: Functional Analysis. Academic Press, New York, 1972.

[59] Rieffel, M.A. Induced representations of $C^{*}$-algebras. Adv. Math. 13: 176-257, 1974.

[60] Rieffel, M.A. Deformation quantization of Heisenberg manifolds. Comm. Math. Phys. 122: $531-562,1989$.

[61] Rieffel, M.A. Quantization and $C^{*}$-algebras. Contemporary Mathematics 167: 66-97, 1994.

[62] Rørdam, M., F. Larsen, and N. Laustsen. An Introduction to K-Theory for $C^{*}$-Algebras. Cambridge University Press, Cambridge, 2000.

[63] Rosenberg, J. $K$-theory of group $C^{*}$-algebras, foliation $C^{*}$-algebras, and crossed products. Contemporary Mathematics 70: 251-301, 1986.

[64] Sjamaar, R. Symplectic reduction and Riemann-Roch formulas for multiplicities. Bull. Amer. Math. Soc. (N.S.) 33: 327-338, 1996. 
[65] Smale, S. Topology and mechanics. I. Invent. Math. 10: 305-331, 1970.

[66] Sundermeyer, K. Constrained Dynamics. Springer-Verlag, Berlin, 1982.

[67] Takahashi, A. A duality between Hilbert modules and fields of Hilbert spaces. Rev. Colombiana Mat. 13: 93-120, 1979.

[68] Taylor, M. Pseudodifferential Operators. Princeton University Press, Princeton, 1982.

[69] Tian, Y. and W. Zhang. An analytic proof of the geometric quantisation conjecture of Guillemin-Sternberg. Invent. Math. 132: 229-259, 1998.

[70] Valette, A. Introduction to the Baum-Connes Conjecture. Birkhäuser, Basel, 2002.

[71] Vergne, M. Quantification géométrique et réduction symplectique. Astérisque 282: 249-278, 2002.

[72] Wegge-Olsen, N.E. K-theory and $C^{*}$-algebras. Oxford University Press, Oxford, 1993.

[73] Weinstein, A. The local structure of Poisson manifolds. J. Diff. Geom. 18: 523-557, 1983.

[74] Wolf, J. Essential self-adjointness for the Dirac operator and its square. Indiana Univ. Math. J. 22: 611-640, 1972.

[75] Xu, P. Morita equivalence of Poisson manifolds. Comm. Math. Phys. 142: 493-509, 1991. 